\title{
Class-Uniformly Resolvable Group Divisible Structures II: Frames.
}

\author{
Peter Danziger* \\ Department of Mathematics, Physics and Computer Science \\ Ryerson Polytechnic University \\ Toronto, ON M5B 2K3, Canada \\ danziger@acs.ryerson.ca \\ Brett Stevens $^{\dagger}$ \\ School of Mathematics and Statistics \\ Carleton University \\ 1125 Colonel By Dr. \\ Ottawa ON K1S 5B6, Canada \\ brett@math.carleton.ca
}

Submitted: Jun 3, 2003; Accepted: Mar 15, 2004; Published: Mar 25, 2004

MR Subject Classifications: 05B05, 05B40

\begin{abstract}
We consider Class-Uniformly Resolvable frames (CURFs), which are group divisible designs with partial resolution classes subject to the class-uniform condition. We derive the necessary conditions, including extremal bounds, build the foundation for general CURF constructions, including a frame variant of the $\lambda$ blow-up construction from part I. We also establish a PBD-closure result. For CURFs with blocks of size two and three we determine the existence of CURFs of type $g^{u}$, completely for $g=3$, with a small list of exceptions for $g=6$, asymptotically for $g=4,5$ and give some other infinite families.
\end{abstract}

\section{Introduction}

A Class-Uniformly Resolvable incidence structure is one where each resolution class has the same number of blocks of each size. Class-uniformly resolvable designs (CURDs) and class-uniformly resolvable group divisible designs (CURGDDs) have been studied in $[7,4,11,14]$, which contain motivations, applications and general discussions of these

*Supported by NSERC discovery grant \#OGP0170220.

${ }^{\dagger}$ Supported by PIMS, MITACS and IBM Watson Research and NSERC.

THE ELECTRONIC JOURNAL OF COMBINATORICS 11 (2004), \#R24 
and related objects. We will assume that the reader is acquainted with the design theory terminology and we refer them to [2].

An idea which has proved productive in the investigation of resolvable structures is that of a frame $[8,13]$. Frames can be generalized to admit class-uniform partial resolutions.

Definition 1.1. A Class-Uniformly Resolvable Frame, $C U R F_{\lambda}$, of type $g^{u}$ with partition $\prod k^{p_{k}}$ is a $\mathrm{GDD}_{\lambda}$ with the additional property that the blocks can be partitioned into partial resolution classes with partition $\prod k^{p_{k}}$ and the complement of a partial resolution class is exactly one group. We say that the class misses the complementary group.

We call such a CURF Homogeneous, HCURF, if the number of partial resolution classes missing each group is a fixed number.

In this definition, and most of the rest of the rest of the paper, we assume that all groups have the same size. If this is not the case, it would be necessary to allow different partitions that correspond to the partial parallel classes missing groups of different sizes. CURFs were first introduced by Lamken et al. [11], where they considered a number of CURD constructions involving CURFs. A CURF with all $g=1$ is a CURD with blocks of size one. If $\lambda=1$ we drop the subscript $\lambda$.

The homogeneous condition is equivalent to requiring that each point in the design is on a fixed number of partial resolution classes. In this case we use $m=r / u$ to denote the number of partial resolution classes missing each group. All of the CURFs that we construct will be homogeneous, but the following example shows that homogeneity is not necessary, even in the case of equal group sizes and only two block sizes.

Example 1.2. A CURF of type $3^{6}$ with partition $2^{6} 3^{1}$, where groups 0,1 and 2 are missed by three classes each, groups 3,4 and 5 are missed by two classes each. The point set will be $\mathbb{Z}_{18}$ and $r_{x}=12$ for points $0 \leq x \leq 8$, whereas $r_{x}=13$ for points $9 \leq x \leq 17$. The groups are defined as follows:

$$
\begin{array}{lll}
\text { group 0: }\{0,1,2\} & \text { group 1: }\{3,4,5\} & \text { group 2: }\{6,7,8\} \\
\text { group 3: }\{9,10,11\} & \text { group 4: }\{12,13,14\} & \text { group 5: }\{15,16,17\}
\end{array}
$$

Each row in Table 1 is a resolution class, the group it misses is indicated on the left. This will be our standard notation throughout this paper.

In Section 2 we derive the necessary conditions for the existence of class-uniformly resolvable frames. When there are only two block sizes we develop tighter conditions and bounds on the admissible sizes of a CURF. In Section 3 we present constructions for CURFs: Wilson's construction, a general multiplicative construction and a construction which allows the reduction of the index. Wilson's construction also provides us with a PBD closure result for CURFs which is notable because closure results have been elusive for CURDs and CURGDDs. In Section 4 we use these constructions to produce HCURFs. 


$\begin{array}{llllllll}0: & \{3,6,9\} & \{7,14\} & \{13,11\} & \{5,12\} & \{16,10\} & \{17,4\} & \{15,8\} \\ 0: & \{4,7,10\} & \{9,14\} & \{8,13\} & \{17,11\} & \{16,6\} & \{3,12\} & \{5,15\} \\ 0: & \{11,12,16\} & \{9,7\} & \{13,15\} & \{14,5\} & \{8,17\} & \{10,3\} & \{4,6\} \\ 1: & \{0,6,12\} & \{15,9\} & \{10,2\} & \{7,11\} & \{16,13\} & \{8,1\} & \{14,17\} \\ 1: & \{1,7,13\} & \{0,10\} & \{12,17\} & \{14,16\} & \{9,8\} & \{15,6\} & \{11,2\} \\ 1: & \{9,13,17\} & \{6,10\} & \{8,16\} & \{14,1\} & \{11,0\} & \{7,12\} & \{15,2\} \\ 2: & \{0,3,15\} & \{2,4\} & \{9,12\} & \{5,16\} & \{10,13\} & \{14,11\} & \{1,17\} \\ 2: & \{2,5,17\} & \{13,3\} & \{4,14\} & \{10,12\} & \{16,0\} & \{1,9\} & \{15,11\} \\ 2: & \{10,14,15\} & \{1,12\} & \{3,17\} & \{4,11\} & \{16,9\} & \{0,5\} & \{2,13\} \\ 3: & \{2,8,14\} & \{0,13\} & \{12,4\} & \{1,15\} & \{7,5\} & \{6,17\} & \{3,16\} \\ 3: & \{1,4,16\} & \{0,14\} & \{8,3\} & \{15,12\} & \{13,5\} & \{2,6\} & \{7,17\} \\ 4: & \{1,5,6\} & \{17,0\} & \{3,11\} & \{10,8\} & \{4,15\} & \{2,9\} & \{16,7\} \\ 4: & \{0,4,8\} & \{1,3\} & \{15,7\} & \{6,11\} & \{16,2\} & \{17,10\} & \{5,9\} \\ 5: & \{2,3,7\} & \{4,13\} & \{9,0\} & \{12,8\} & \{10,5\} & \{11,1\} & \{14,6\} \\ 5: & \{5,8,11\} & \{12,2\} & \{4,9\} & \{7,0\} & \{3,14\} & \{1,10\} & \{13,6\}\end{array}$

Table 1: A non-homogeneous CURF of type $3^{6}$ with partition $2^{6} 3^{1}$.

\section{Necessary Conditions}

\subsection{General Necessary Conditions}

We now give the general necessary conditions for the existence of a $\mathrm{CURF}_{\lambda}$ of type $g^{u}$ with partition $\prod k^{p_{k}}$ containing $r$ partial resolution classes, in which the $i$ th group, $G_{i}$ is missed by $m_{i}$ resolution classes. Standard counting arguments give the following:

$$
\begin{gathered}
u \geq \max _{k \in K}\{k\}+1, \\
g(u-1)=\sum_{k \in K} k p_{k}, \\
r \sum_{k \in K} k(k-1) p_{k}=\lambda u(u-1) g^{2}, \\
r=\sum_{i=1}^{u} m_{i} .
\end{gathered}
$$

If the CURF is homogeneous then each group is missed by $m=r / u$ partial resolution classes and Equation 3 reduces to

$$
m \sum_{k \in K} k(k-1) p_{k}=\lambda(u-1) g^{2} .
$$

Let $r_{k}(x)$ be the number of blocks of size $k$ which contain the point $x$. It is not generally true that $r_{k}$ is independent of $x$ as illustrated in Example 1.2. The following example illustrates that even homogeneity is not sufficient to guarantee independence of $r_{k}(x)$ from $x$. 
Example 2.1. The following is an $\mathrm{HCURF}_{8}$ of type $1^{9}$ with partition $1^{3} 2^{1} 3^{1}$. Each group is missed by 8 partial resolution classes. The point set is $\mathbb{Z}_{8} \cup\{\infty\}$. Consider the following 9 partial resolution classes. Develop the classes additively modulo 8.

$\begin{array}{clllll}\infty: & \{0,1,3\} & \{4,5\} & \{2\} & \{6\} & \{7\} \\ 2: & \{0,1,3\} & \{4,5\} & \{6\} & \{7\} & \{\infty\} \\ 2: & \{0,1,3\} & \{4,5\} & \{6\} & \{7\} & \{\infty\} \\ 5: & \{0,1,3\} & \{2,4\} & \{6\} & \{7\} & \{\infty\} \\ 5: & \{0,1,3\} & \{2,4\} & \{6\} & \{7\} & \{\infty\} \\ 0: & \{\infty, 3,7\} & \{2,4\} & \{1\} & \{5\} & \{6\} \\ 1: & \{\infty, 3,7\} & \{2,5\} & \{1\} & \{4\} & \{6\} \\ 1: & \{\infty, 3,7\} & \{2,5\} & \{1\} & \{4\} & \{6\} \\ 1: & \{\infty, 3,7\} & \{2,5\} & \{1\} & \{4\} & \{6\}\end{array}$

This example has high $\lambda$, but it satisfies the hypotheses of Theorem 3.4 and so can be used to produce an $\mathrm{HCURF}_{1}$ of type $8^{9}$ with partition $1^{24} 2^{8} 3^{8}$ which contains points that appear in no pairs.

We call a CURF point-block regular if every point appears the same number of times in blocks of each size, i.e. $r_{k}(x)=r_{k}$ is constant across $x$. A CURF is called point regular if all points are in the same number of blocks. Counting the number of blocks that contain a point $x$ yields

$$
\sum_{k \in K} r_{k}(x)=r-m_{i}
$$

where $x$ is in the group $G_{i}$. Thus the condition of homogeneity, $m_{i}=m$ for every $i$, is equivalent to point regularity. Further, if a CURF is point-block regular then it is homogeneous. The falsity of the converse is demonstrated by Example 2.1. Counting the number of pairs containing $x$ in every block through $x$ gives other necessary conditions:

$$
\sum_{k \in K} r_{k}(x)(k-1)=\lambda g(u-1) .
$$

Assuming that there are no blocks of size 1 and our block sizes are enumerated in order, we get

$$
k_{1}-1 \leq \frac{\lambda g(u-1)}{r-m_{i}} \leq k_{n}-1 .
$$

Taking $m_{\min }$ and $m_{\max }$ to be the minimum and maximum $m_{i}$ 's respectively and $\bar{m}$ to be the average of the $m_{i}$ 's, we have

Theorem 2.2. If $m_{\min }>0$ and $n>1$ then there is no CURF $F_{1}$ of type $g^{u}$ with partition $\prod_{i} k_{i}^{p_{k_{i}}}, 1<k_{1}<\ldots<k_{n}$, for any $g<k_{1}$.

Proof. Suppose that such a CURF exists. We first consider the case $g<k_{1}-1$, Equation 8 gives $m_{\min } \leq g /\left(k_{1}-1\right)<1$. 
When $g=k_{1}-1$, Equation 8 then gives $1 \leq m_{\min } \leq \bar{m} \leq g /\left(k_{1}-1\right)=1$, so $m_{\text {min }}=\bar{m}=1$. Thus $m_{i}=1$ for all $i$ and the CURF is homogeneous and so Equations 2 and 5 give

$$
\sum_{i} k_{i}\left(k_{i}-1\right) p_{k_{i}}=\left(k_{1}-1\right) \sum_{i} k_{i} p_{k_{i}} .
$$

But since there are at least two block sizes and $k_{1}$ is the smallest block size

$$
\sum_{i} k_{i}\left(k_{i}-1\right) p_{k_{i}}>\left(k_{1}-1\right) \sum_{i} k_{i} p_{k_{i}}
$$

The existence of $k$-frames with group size $k-1$ demonstrates the necessity of at least two block sizes in the hypotheses of the theorem. Example 2.3 illustrates that the condition $m_{\min }>0$ is indeed necessary. Example 2.4 illustrates that the bound $g<k_{1}$ is the strongest possible.

Example 2.3. A CURF of type $1^{25}$ with partition $2^{9} 3^{2}$, with 20 resolution classes, the

\begin{tabular}{|c|c|c|c|c|c|c|c|c|c|c|}
\hline$\{22$ & $20\}$ & $21\}$ & $4\}$ & $\{3,10\}$ & $\{14,18\}$ & $\{16,19\}$ & $\{24,13\}$ & $\{17,23\}$ & $\{5,7\}$ & $\{0,2\}$ \\
\hline 6,3$\}$ & $\{9,4,20\}$ & $\{12,22\}$ & $\{24,23\}$ & $\{0,5\}$ & $\{13,6\}$ & $\{14,17\}$ & $\{10,21\}$ & $\{1,19\}$ & $\{7,15\}$ & $\{8,18\}$ \\
\hline$\{2,13,4\}$ & $\{23,15,18\}$ & $\{20,21\}$ & $\{6,10\}$ & $\{8,12\}$ & $\{11,19\}$ & $\{9,17\}$ & $\{24,7\}$ & $\{16,14\}$ & $\{0,1\}$ & $\{22,5\}$ \\
\hline$\{5,12,6\}$ & $\{11,9,1\}$ & $\{8,24\}$ & $\{18,3\}$ & $\{17,2\}$ & $\{2019\}$ & $\{7,14\}$ & $\{15,22\}$ & $\{13,16\}$ & $\{21,23\}$ & $\{10,0\}$ \\
\hline$\{4,12,3\}$ & $\{10,19,2\}$ & $\{8,16\}$ & $\{6,18\}$ & $\{9,15\}$ & $\{11,13\}$ & $\{20,0\}$ & $\{24,22\}$ & $\{21,14\}$ & $\{17,1\}$ & $\{23,7\}$ \\
\hline$\{8,14,19\}$ & $\{5,18,4\}$ & $\{7,9\}$ & $\{13,20\}$ & $\{1,15\}$ & $\{3,17\}$ & $\{11,22\}$ & $\{16,2\}$ & $\{10,12\}$ & $\{0,23\}$ & $\{21,24\}$ \\
\hline$\{9,21,19\}$ & $10,20\}$ & $\{11,2\}$ & $14\}$ & $\{8,13\}$ & $\{0,15\}$ & $\{1,22\}$ & $\{16,5\}$ & $\{3,24\}$ & $23\}$ & $\{6,17\}$ \\
\hline$\{24,0,11\}$ & 18 & $\{6,23\}$ & $14\}$ & $\{7,20\}$ & $2\}$ & $\{5,21\}$ & $\{4,22\}$ & & $19\}$ & $13\}$ \\
\hline$\{1,6,14\}$ & $\{18,24,17\}$ & $\{12,13\}$ & $\{10,11\}$ & $\{21,4\}$ & $\{23,20\}$ & $\{19,5\}$ & $\{0,16\}$ & $15\}$ & $\{8,2\}$ & $\{22,7\}$ \\
\hline 1,11$\}$ & $\{22,0,13\}$ & $\{9,24\}$ & $\{2,3\}$ & $\{12,1\}$ & $\{14,5\}$ & $\{18,19\}$ & $\{6,16\}$ & $\{8,23\}$ & $\{17,20\}$ & $\{15,4\}$ \\
\hline$\{4,10,23\}$ & $\{20,1,5\}$ & $\{19,13\}$ & $\{14,9\}$ & $\{6,0\}$ & $\{18,21\}$ & $\{12,24\}$ & $\{2,7\}$ & $\{16,22\}$ & $\{15,17\}$ & $\{3,8\}$ \\
\hline$\{22,23,19\}$ & $\{2,20,6\}$ & $\{24,10\}$ & $\{17,13\}$ & $\{9,16\}$ & $\{11,14\}$ & $\{15,5\}$ & $\{1,8\}$ & $\{21,3\}$ & $\{7,18\}$ & $\{0,4\}$ \\
\hline & $\{14,3,0\}$ & $\{15,10\}$ & $\{9,23\}$ & $\{4$ & $\{7,12\}$ & $6\}$ & $\{21 \quad 1\}$ & $19\}$ & $22\}$ & 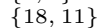 \\
\hline$\{24,15,16\}$ & $\{7,10,17\}$ & $\{12,2\}$ & $\{1,18\}$ & $\{20,22\}$ & $\{0,19\}$ & $\{9,5\}$ & $\{11,23\}$ & $\{4,8\}$ & $\{3,6\}$ & $13,21\}$ \\
\hline$\{17,12,11\}$ & $\{8,6,7\}$ & $\{9,0\}$ & $\{19,24\}$ & $\{3,5\}$ & $\{16,4\}$ & $\{10,1\}$ & $\{22,21\}$ & $\{14,20\}$ & $\{2,18\}$ & $\{23,13\}$ \\
\hline & $\{15,13,14\}$ & $\{0,18\}$ & & $\{23,2\}$ & $\{9,12\}$ & $\{22,10\}$ & $\{6,21\}$ & & $\{3,19\}$ & $\{11,20\}$ \\
\hline$\{1,7,16\}$ & $\{9,13,10\}$ & $\{18,22\}$ & $5156\}$ & $\{2,21\}$ & $\{12,0\}$ & $\{3,20\}$ & $\{23,5\}$ & $\{11,8\}$ & $\{14,24\}$ & $\{4,19\}$ \\
\hline$\{14,22,2\}$ & $\{1,23,3\}$ & $\{11,6\}$ & $\{20,12\}$ & $\{4,24\}$ & $\{8,9\}$ & $\{17,0\}$ & $\{10,16\}$ & $\{7,19\}$ & $\{13,5\}$ & $\{21,15\}$ \\
\hline & $\{8,0,21\}$ & $\{1,2\}$ & $\{4,6$ & $\{14,12\}$ & $\{20,24\}$ & $\{11,15\}$ & $\{17,22\}$ & $\{16,23\}$ & $\{9,18\}$ & $\{5,10\}$ \\
\hline 19,12$\}$ & $\{21,16,17\}$ & $\{13,18\}$ & $\{23,14\}$ & $\{24,6\}$ & $\{3,22\}$ & $\{9,2\}$ & $\{1,4\}$ & $\{8,10\}$ & $\{5,11\}$ & $\{7,0\}$ \\
\hline
\end{tabular}
groups are individual points. The group $\{0\}$ is missed by no partial resolution class.

Example 2.4. A CURF of type $4^{15}$ with partition $2^{16} 3^{8}$. One group has $m_{i}=14$ and all other groups are missed by exactly one partial resolution class. Start with a $\mathrm{CURF}_{2}$ of type $1^{15}$ with partition $2^{4} 3^{2}$, with 21 resolution classes. The point set is $\mathbb{Z}_{7} \times \mathbb{Z}_{2} \cup\{\infty\}$. Develop the following resolution classes modulo 7 in the first coordinate.

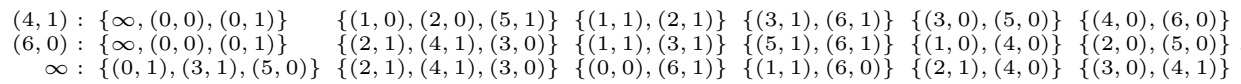

$\mathrm{A} \mathrm{CURF}_{4}$ satisfying the conditions of Theorem 3.4 is obtained by doubling these blocks and so produces the desired $\mathrm{CURF}_{1}$.

\subsection{Necessary Conditions for CURFs with 2 Block Sizes}

In this section we consider the case where there are exactly two block sizes $k$ and $l$; we will consider $k$ and $l$ to be interchangeable. The bounds here are derived in a similar manner to those in [4]. 


$$
\begin{aligned}
l p_{l} & \equiv g(u-1) \bmod k \\
r_{l}(x) & =\frac{\lambda g(u-1)-\left(r-m_{i}\right)(k-1)}{l-k} .
\end{aligned}
$$

Thus for two block sizes, homogeneity implies point-block regularity and thus the two are equivalent (see Equation 6). To illustrate that these conditions do not generalize, Example 1.2 is a CURF with two block sizes which is neither homogeneous nor point-block regular. Example 2.1 gives an HCURF with three block sizes which is not point-block regular.

Letting $\alpha_{l}=l(l-k) p_{l}$ and defining $d=\operatorname{gcd}\left(r(k-1)^{2}, \lambda\left((k-1) g u-\alpha_{l}\right)\right)$, we get

$$
g(u-1) \leq \lambda\left|\frac{\alpha_{l}}{d}\left(\frac{\alpha_{l}}{k-1}-g\right)\right|-\frac{\alpha_{l}}{k-1}
$$

When $g(k-1)=\alpha_{l}$, which requires that $l>k$, we obtain no bound on $g(u-1)=$ $r(k-1) / \lambda$. When the CURF is homogeneous, and defining $d=\operatorname{gcd}(m(u-1)(k-$ $\left.1)^{2}, \lambda\left((k-1) g(u-1)-\alpha_{l}\right)\right)$, we get

$$
g(u-1) \leq \frac{\alpha_{l}\left(\lambda \alpha_{l}-d\right)}{d(k-1)}
$$

When the blocks are of size 2 and 3 we get

$$
\begin{aligned}
p_{3} & \equiv g(u-1) \bmod 2, \\
2 p_{2} & \equiv g(u-1) \bmod 3, \\
\lambda p_{3} & \equiv 0 \bmod 2, \\
r_{3}(x) & =\lambda g(u-1)-r+m_{i}, \\
r_{2}(x) & =2\left(r-m_{i}\right)-\lambda g(u-1),
\end{aligned}
$$

and assuming homogeneity since it is the most useful case,

$$
\begin{aligned}
& g(u-1) \leq 3 p_{3}\left(\lambda \frac{3 p_{3}}{d_{3}}-1\right) \\
& g(u-1) \leq p_{2}\left(\lambda \frac{p_{2}}{d_{2}}+1\right)
\end{aligned}
$$

where $d_{3}=\operatorname{gcd}\left(m(u-1), \lambda\left(g(u-1)-3 p_{3}\right)\right)$ and $d_{2}=\operatorname{gcd}\left(2 m(u-1), \lambda\left(g(u-1)+p_{2}\right)\right)$.

\section{General Frame Constructions}

In this section we give some general constructions for creating CURFs. 
Theorem 3.1 (Wilson's Fundamental Frame Construction). If there exists a $K$ $G D D$ of type $g^{u}$, and for every $k \in K$ there exists an (H)CURF of type $n^{k}$ with partition $\prod i^{(k-1) q_{i}}$ (which implies a constant ratio $r / k$ ), then there exists an (H)CURF of type $(n g)^{u}$ and partition $\prod i^{(u-1) q_{i}}$.

Proof. This is Wilson's fundamental frame construction [8]. We blow up the master $K$ GDD giving each point weight $n$. Place the ingredient $(\mathrm{H}) \mathrm{CURF}$ s on the blown up blocks. We recover the partial parallel classes missing the group $G \times \mathbb{Z}_{n}$, where $G$ is a group of the master $K$-GDD, by considering the blocks of the master design through each point $x \in G$. The union of one partial parallel class missing $\{x\} \times \mathbb{Z}_{n}$ from each of the the frames placed on the blow up of these blocks form a partial parallel class missing $G \times \mathbb{Z}_{n}$.

This theorem gives a PBD closure result for CURFs. For a fixed $\bar{m}$ and $g$, modular conditions for admissible $u$ can be found. There is another variation of Wilson's Construction.

Theorem 3.2. If there exists an (H)CURF of type $g^{u}$ with partition $\prod k^{p_{k}}$ and there exists an CURGDD of type $n^{k}$ for each $k$ that appears with partition $\prod i^{q_{i, k}}$, each with the same $r$ then there exists an (H)CURF of type $(n g)^{u}$ with partition $\prod i^{\sum_{k} q_{i, k} p_{k}}$.

Proof. Blow up each point of the master CURF by $n$ and fill the blown up blocks with the ingredient CURGDDs [8].

We now include a standard construction for breaking up the groups of a CURF.

Theorem 3.3 (Breaking up Groups). If there exists an CURF of type $(n g)^{u}$ with partition $\prod k^{p_{k}}$ with $r$ partial resolution classes and for each group $G_{i}$ there is an CURF of type $g^{n}$ and partition $\prod k^{q_{k}}$ with $m_{i}$ resolution classes, then there exists an CURF of type $g^{n u}$ with partition $\prod k^{p_{k}+q_{k}}$.

Proof. Replace groups by smaller structure.

We now present a notable construction which allows us to reduce the index of a $\mathrm{CURF}_{\lambda}$, whilst increasing the group size, similar to that found in [7]. We refer the reader there for definitions, notation and proof. The details of checking that the frame property is preserved are straightforward.

Theorem 3.4 ( $\lambda$ Blow-up). If there exists a $(H) C U R F_{\lambda}$ of type $g^{u}$ with partition $\prod k^{p_{k}}$ 1-maximally contained in $(X, \mathcal{M})$ and for each $M \in \mathcal{M}$ there exists an $\operatorname{RTD}_{\lambda_{1}}(|M|, \lambda)$ then there exists a $(H) C U R F_{\lambda_{1}}$ of type $(\lambda g)^{u}$ and partition $\prod k^{\lambda p_{k}}$.

When the only block sizes in $\mathcal{B}$ are 1,2 and $k$ and the blocks of size $k$ (repetitions ignored) form a packing of index 1 then the hypotheses of Theorem 3.4 are satisfied. We illustrate this construction with the following example. 
Example 3.5. We present an $\mathrm{HCURF}_{10}$ of type $1^{8}$ with partition $2^{2} 3^{1}$ with $r=56$ $(m=7)$ resolution classes. The blocks of size 3 form the blocks of a packing on 8 points repeated seven times. Develop the following seven base blocks cyclically over $\mathbb{Z}_{8}$.

$\begin{array}{llll}5: & \{0,1,3\} & \{2,6\} & \{4,7\} \\ 5: & \{0,1,3\} & \{2,6\} & \{4,7\} \\ 5: & \{0,1,3\} & \{2,6\} & \{4,7\} \\ 4: & \{0,1,3\} & \{2,6\} & \{5,7\} \\ 7: & \{0,1,3\} & \{2,6\} & \{4,5\} \\ 7: & \{0,1,3\} & \{2,4\} & \{5,6\} \\ 7: & \{0,1,3\} & \{2,4\} & \{5,6\}\end{array}$

We take

$$
\begin{aligned}
\mathcal{M}= & \{\{0,1,3\},\{1,2,4\},\{2,3,5\},\{3,4,6\},\{4,5,7\},\{5,6,0\}, \\
& \{6,7,1\},\{7,0,2\},\{0,4\},\{1,5\},\{2,6\},\{3,7\}\} .
\end{aligned}
$$

Applying Theorem 3.4 produces an $\mathrm{HCURF}_{1}$ of type $10^{8}$ with partition $2^{20} 3^{10}$.

\section{Existence of HCURFs}

We now demonstrate the utility of these constructions. For blocks of size 2 and 3 , we use PBD closure to establish the existence of all HCURFs with $g=3$, asymptotic existence $g=4,5$, all but a small finite number for $g=6$ for all $m$ and $g=7$ for $m=4$ and asymptotically for $g=8$ for $m=7$. Following this, we apply Theorems 3.1 and 3.2 with different kinds of ingredients. Finally we end with some prime power difference constructions. We will make frequent use of Theorem 3.4 in these solutions.

\subsection{PBD Closure Constructions}

For block sizes 2 and 3 if either $p_{2}$ or $p_{3}$ are zero then the HCURFs are 2-frames or 3frames respectively and their existence has been completely solved [2]. Therefore, in what follows we will assume that $p_{2}, p_{3}>0$. For each $g$ there is a range of possible values of $m$, obtained from Equation 8. for each of these values of $m$ we obtain modular conditions on $u$ which are amenable to PBD-closure. For each value of $g$, Theorem 3.2 with RTDs as ingredients give existence results for HCURFs with group size $g n$ for any $n \neq 2,6$.

\subsection{1 $g=2$}

If $g=2$ there are no HCURFs with $p_{2}, p_{3}>0$ by Equation 8 .

\subsection{2 $g=3$}

When $g=3$ the admissible value of $m$ that permits both block sizes is $m=2$. In this case the HCURFs have parameters $3^{4 t+1}$ and partition $2^{3 t} 3^{2 t}$ for $t>0$; we solve this case completely. 
Theorem 4.1. An HCURF of type $(3 n)^{u}$ with $u=4 t+1$ partition $2^{3 t} 3^{2 t}$, for all $n \neq 2,6$ and all $u$.

Proof. ¿From the equations in Section 2, the conditions are necessary. In particular Inequality 8 implies that $m=2$. The case $m=3$ corresponds to a 2 -frame $\left(p_{3}=0\right)$. We now establish the existence for $m=2$ and $u \equiv 1 \bmod 4$.

In [9] (see also [2]) it is shown that $\{5,9,13,17,29,33\}$ is a generating set for $\{x \mid x \in$ $\mathbb{Z}, x>4, x \equiv 1 \bmod 4\}$. Thus there is a PBD with block sizes from $\{5,9,13,17,29,33\}$ on $v$ points for every integer $v \equiv 1 \bmod 4$, larger than 4 . Thus by establishing the existence of HCURFs of type $3^{u}$ for $u \in\{5,9,13,17,29,33\}$ and applying Theorem 3.1, giving each point weight 3 , we produce all the desired HCURFs.

When $u \equiv 1,5$ mod 12 we construct HCURFs directly using a difference construction. The point set is $\mathbb{Z}_{3} \times \mathbb{Z}_{u}$, the groups are $\{(0, i),(1, i),(2, i)\}$ for $0 \leq i<u$. Consider the following two partial classes, each missing the group $\{(0,0),(1,0),(2,0)\}$. The first class is

$$
\begin{array}{ll} 
\pm\{(0, i),(1,2 i),(2,3 i)\} & 1 \leq i \leq \frac{u-1}{4} \\
\{\{(0, i),(0,-i)\},\{(1,2 i),(1,-2 i)\},\{(2,3 i),(2,-3 i)\}\} & \frac{u+3}{4} \leq i \leq \frac{u-1}{2} .
\end{array}
$$

The second class is

$$
\begin{array}{ll} 
\pm\{(0, i),(1,2 i),(2,3 i)\} & \frac{u+3}{4} \leq i \leq \frac{u-1}{2} \\
\{\{(0, i),(0,-i)\},\{(1,2 i),(1,-2 i)\},\{(2,3 i),(2,-3 i)\}\} & 1 \leq i \leq \frac{u-1}{4} .
\end{array}
$$

Develop these classes modulo $u$ to get all the classes. In particular, this constructs HCURFs for $u=5,13,17$ and 29 .

We now present an HCURF of type $3^{9}$ with partition $2^{6} 3^{4}$. The point set is $\mathbb{Z}_{3} \times \mathbb{Z}_{9}$ and the groups are $\{(0, i),(1, i),(2, i)\}$ for $0 \leq i \leq 8$. Develop the following classes, which miss the group $\{(0,6),(1,6),(2,6)\}, \bmod (-, 9)$.

$\{(0,0),(1,1),(2,2)\},\{(0,1),(1,3),(2,5)\},\{(0,2),(1,5),(2,8)\},\{(0,4),(1,8),(2,3)\}$, $\{(0,3),(0,5)\},\{(0,7),(0,8)\},\{(1,0),(1,2)\},\{(1,4),(1,7)\},\{(2,0),(2,4)\},\{(2,1),(2,7)\}$

$\{(0,0),(1,5),(2,1)\},\{(0,1),(1,7),(2,4)\},\{(0,2),(1,0),(2,7)\},\{(0,4),(1,3),(2,2)\}$, $\{(0,3),(0,7)\},\{(0,5),(0,8)\},\{(1,1),(1,2)\},\{(1,4),(1,8)\},\{(2,0),(2,8)\},\{(2,3),(2,5)\}$

We now present an HCURF of type $3^{33}$ with partition $2^{24} 3^{16}$. The point set is $\mathbb{Z}_{3} \times \mathbb{Z}_{33}$ and the groups are $\{(0, i),(1, i),(2, i)\}$ for $0 \leq i \leq 32$. Develop the following classes, which miss the group $\{(0,18),(1,18),(2,18)\}, \bmod (-, 33)$.

$\{(0,0),(1,1),(2,2)\},\{(0,1),(1,3),(2,5)\},\{(0,2),(1,5),(2,8)\},\{(0,3),(1,7),(2,11)\}$, $\{(0,4),(1,9),(2,14)\},\{(0,5),(1,11),(2,17)\},\{(0,6),(1,13),(2,20)\}$, $\{(0,7),(1,15),(2,23)\},\{(0,8),(1,17),(2,26)\},\{(0,9),(1,19),(2,29)\}$, $\{(0,10),(1,21),(2,32)\},\{(0,12),(1,24),(2,3)\},\{(0,13),(1,26),(2,6)\}$, $\{(0,14),(1,28),(2,9)\},\{(0,15),(1,30),(2,12)\},\{(0,16),(1,32),(2,15)\}$, $\{(0,11),(0,22)\},\{(0,17),(0,31)\},\{(0,19),(0,32)\},\{(0,20),(0,27)\},\{(0,21),(0,30)\}$, $\{(0,23),(0,28)\},\{(0,24),(0,26)\},\{(0,25),(0,29)\},\{(1,0),(1,6)\},\{(1,2),(1,10)\}$, $\{(1,4),(1,8)\},\{(1,12),(1,23)\},\{(1,14),(1,31)\},\{(1,16),(1,29)\},\{(1,20),(1,27)\}$, 
$\{(1,22),(1,25)\},\{(2,0),(2,1)\},\{(2,4),(2,7)\},\{(2,10),(2,16)\},\{(2,13),(2,21)\}$,

$\{(2,19),(2,28)\},\{(2,22),(2,24)\},\{(2,25),(2,30)\},\{(2,27),(2,31)\}$

$\{(0,0),(1,17),(2,1)\},\{(0,1),(1,19),(2,4)\},\{(0,2),(1,21),(2,7)\}$,

$\{(0,3),(1,23),(2,10)\},\{(0,4),(1,25),(2,13)\},\{(0,5),(1,27),(2,16)\}$,

$\{(0,6),(1,29),(2,19)\},\{(0,7),(1,31),(2,22)\},\{(0,8),(1,0),(2,25)\}$,

$\{(0,9),(1,2),(2,28)\},\{(0,10),(1,4),(2,31)\},\{(0,12),(1,7),(2,2)\}$,

$\{(0,13),(1,9),(2,5)\},\{(0,14),(1,11),(2,8)\},\{(0,15),(1,13),(2,11)\}$,

$\{(0,16),(1,15),(2,14)\},\{(0,11),(0,27)\},\{(0,17),(0,32)\},\{(0,19),(0,31)\}$,

$\{(0,20),(0,30)\},\{(0,21),(0,29)\},\{(0,22),(0,28)\},\{(0,23),(0,26)\},\{(0,24),(0,25)\}$,

$\{(1,1),(1,10)\},\{(1,3),(1,8)\},\{(1,5),(1,6)\},\{(1,12),(1,26)\},\{(1,14),(1,32)\}$,

$\{(1,16),(1,28)\},\{(1,20),(1,30)\},\{(1,22),(1,24)\},\{(2,0),(2,12)\},\{(2,3),(2,20)\}$,

$\{(2,6),(2,26)\},\{(2,9),(2,24)\},\{(2,15),(2,29)\},\{(2,17),(2,27)\},\{(2,21),(2,32)\}$,

$\{(2,23),(2,30)\}$

We have constructed an HCURF of type $3^{u}$ with partition $2^{3(u-1) / 4} 3^{u-1 / 2}$ for each $u \in\{5,9,13,17,29,33\}$ hence the result follows from Theorem 3.1.

\subsection{3 $g=4$}

We now consider the case where $g=4$. The admissible value of $m$ that permits both block sizes is $m=3$. In this case the HCURFs have parameters $4^{9 t+1}$ and partition $2^{12 t} 3^{4 t}$ for $t>0$.

Theorem 4.2. There exists an HCURF of type $(4 n)^{u}$ with $u=9 t+1$ partition $2^{12 n t} 3^{4 n t}$ for all $u$ of the form

$$
u=\prod_{m \in D} m^{e_{m}}
$$

where $D$ is the set of integers defined below, and for all $u \equiv 1 \bmod 9$ sufficiently large.

Proof. Let $C=\{9 t+1 \mid 1 \leq t \leq 100\}$, in [5] it is shown that an $\mathrm{HCURF}_{4 n}$ of type $1^{u}$ partition $2^{(u-1) / 3} 3^{(u-1) / 9}$ exists satisfying the conditions of Theorem 3.4 for all $u \in C$, the first 5 are given in the appendix. Let $A=\left\{x^{2}+x+1 \mid x \in C, x=q+1, q\right.$ a prime power $\}$ and let $B=\left\{x^{2} \mid x \in C, x=q, q\right.$ a prime power $\}$. Using the projective and affine planes in Theorem 3.1 we get an HCURF of type $4^{u}$ for $u \in A \cup B$. Let $D=A \cup B \cup C$, by repeated application of Theorems 3.2 and 3.3 we get the first result.

By Wilson's theorem the necessary conditions, $v \equiv 1 \bmod 9$, for the existence of a $\operatorname{PBD}(v,\{10,19\}, 1)$ are asymptotically sufficient [15]. Applying Theorem 3.1 gives the asymptotic result.

\subsection{4 $g=5$}

For $g=5$ there are two values: $m=3,4$; we show their asymptotic existence in order.

Theorem 4.3. There exists an HCURF of type $(5 n)^{u}$ with $u=18 t+1$, partition $2^{15 n t} 3^{20 n t}$ for all $n \neq 2,6, u=19^{e} 37^{f} 55^{g}$ and asymptotically for all $u \equiv 1 \bmod 18$. 
Proof. For $t=1,2,3$ the HCURFs are given in Appendix A.2. Repeated application of Theorems 3.2 and 3.3 gives the first result. By Wilson's theorem the necessary conditions for the existence of a $\operatorname{PBD}(v,\{19,37,55\}, 1), v \equiv 1 \bmod 18$, are asymptotically sufficient [15]. Applying Theorem 3.1 gives the result.

Similarly the HCURFs given in Appendix A.3 give

Theorem 4.4. There exists an HCURF of type $(5 n)^{u}$ with $u=24 t+1$, partition $2^{45 n t} 3^{10 n t}$ for all $n \neq 2,6, u=25^{e} 49^{f}$ and asymptotically for all $u \equiv 1 \bmod 24$.

\subsection{5 $g=6$}

For $g=6$ there are two values: $m=4,5$; in each case we find all but a small number of exceptions.

Theorem 4.5. There exists an HCURF of type $(6 n)^{u}$ with $u=2 t+1$, partition $2^{3 n t} 3^{2 n t}$ for all $n \neq 2,6$,

$u \notin\{43,51,59,71,75,83,87,95,107,111,115,119,131,135,139,167,179,183,191,195\}$.

Proof. By PBD closure [1], it is sufficient to find HCURFs for $u \in\{5,7,9,11,13\}$. We present the first of these here, the remaining are given in Appendix A.4.

The point set is $\mathbb{Z}_{6} \times \mathbb{Z}_{5}$. The following class misses the group $\{(i, 4) \mid 0 \leq i \leq 5\}$ :

$$
\begin{aligned}
& \{(0,0),(1,1),(2,2)\}\{(0,2),(0,3),(3,0)\}\{(4,0),(5,1)\}\{(3,1),(5,3)\}\{(3,2),(4,3)\} \\
& \{(1,2),(1,3),(5,0)\}\{(2,1),(2,3),(4,2)\}\{(0,1),(5,2)\}\{(1,0),(4,1)\}\{(2,0),(3,3)\} \text { • }
\end{aligned}
$$

Multiply this class by $(1,1),(-1,1),(1,2)$, and $(-1,2)$ and develop the resulting classes modulo $(-, 5)$.

In [6] the existence of an HCURF of type $6^{5 t+1}$ partition $2^{12 t} 3^{2 t}$ for $5 t+1 \in\{6,11,16$, $21,26,36,41\}$ is established. These values represent a generating set for $u \equiv 1 \bmod 5$ [2] with 14 exceptions, six of which are also in [6]. This leaves $u \in\{56,86,116,191,236,291$, $311,321\}$ open. Thus we may apply Theorem 3.1 and Corollary 3.2 to give the following, the case $n=6$ is given in [11].

Theorem 4.6. There exists an HCURF of type $(6 n)^{u}$ with $u=5 t+1$ partition $2^{12 n t} 3^{2 n t}$ for every $n \neq 2$, with 8 possible exceptions in $u$.

\subsection{6 $g=7$}

For $g=7, m=4,5,6$ are possible; we show existence for the family $m=4$ using the PBD-closure results known for $u \equiv 1 \bmod 8$ [2].

Theorem 4.7. There exists an HCURF of type $(7 n)^{u}$ with $u=8 t+1$, partition $2^{7 n t} 3^{14 n t}$ for all $n \neq 2,6$, with 66 possible exceptions in $u$.

Proof. Theorem 4.14 gives $u=9$. In Appendix A.5 we give these HCURFs for $u \in$ $\{17,25,33,41,49,57,65,89,97,105,113,121,129\}$. This corresponds to the generating set from [2] (p. 212) for $1 \bmod 8$, we refer the reader there for a list of the 66 possible exceptions. 


\subsection{7 $g=8$}

For $g=8, m=5,6,7$ are possible; we show asymptotic existence for the family $m=7$.

Theorem 4.8. There exists an HCURF of type $(8 n)^{u}$ with $u=21 t+1$, partition $2^{72 t n} 3^{8 t n}$ and $m=7 n$, for all $n \geq 1$ and $u=22^{e} 43^{f}$ and asymptotically for all $u \equiv 1 \bmod 21$.

Proof. For $u \in\{22,43\}$ we present an $\mathrm{HCURF}_{8}$ of type $1^{u}$ with partition $2^{9(u-1) / 21} 3^{(u-1) / 21}$ which satisfies the conditions of Theorem 3.4 in Appendix A.6. For each $n$ apply Theorem 3.4 to $n$ copies of the HCURF. Then by repeated application of Theorems 3.2 and 3.3 we get the result. By Wilson's theorem the necessary conditions for the existence of a $\operatorname{PBD}(v,\{22,43\}, 1), v \equiv 1 \bmod 21$, are asymptotically sufficient [15]. Applying Theorem 3.1 gives the result.

\subsection{Product Constructions}

Up to this point we have used closure results, we now give some other types of existence proofs. Since the existence of 4-GDDs has been completely solved finding just one HCURF with $u=4$ produces spectacular results.

Lemma 4.9. [10] Let $g$ and $u$ be positive integers. Then there exists a 4-GDD of type $g^{u}$ if and only if the following conditions are satisfied:

- $g \equiv 1,5 \bmod 6, u \equiv 1,4 \bmod 12$ and $u \geq 4$;

- $g \equiv 2,4 \bmod 6, u \equiv 1 \bmod 3, u \geq 4$ and $(g, u) \neq(2,4)$;

- $g \equiv 3 \bmod 6, u \equiv 0,1 \bmod 4$ and $u \geq 4 ;$ and

- $g \equiv 0 \bmod 6, u \geq 4$ and $(g, u) \neq(6,4)$;

Lemma 4.10. There exists an HCURF of type $12^{4}$ with partition $2^{12} 3^{4}$.

Proof. We exhibit an $\mathrm{HCURF}_{4}$ of type $3^{4}$ with partition $2^{3} 3^{1}$ satisfying the conditions of Theorem 3.4. The set of point is $\mathbb{Z}_{2} \times \mathbb{Z}_{2} \times \mathbb{Z}_{3}$. The groups are $\{i\} \times \mathbb{Z}_{3}$ where $i \in \mathbb{Z}_{2} \times \mathbb{Z}_{2}$. Develop the following three partial parallel classes over $\mathbb{Z}_{2} \times \mathbb{Z}_{2} \times \mathbb{Z}_{3}$.

$$
\begin{array}{llll}
\{(0,1,2),(1,0,1),(1,1,0)\} & \{(0,1,1),(1,0,2)\} & \{(0,1,0),(1,1,2)\} & \{(1,0,0),(1,1,1)\} \\
\{(0,1,2),(1,0,1),(1,1,0)\} & \{(0,1,1),(1,1,1)\} & \{(0,1,0),(1,0,0)\} & \{(1,0,2),(1,1,2)\} . \\
\{(0,1,2),(1,0,1),(1,1,0)\} & \{(0,1,1),(1,1,1)\} & \{(0,1,0),(1,0,0)\} & \{(1,0,2),(1,1,2)\}
\end{array} .
$$

Theorem 4.11. Let $g$ and $u$ be positive integers. Then there exists an HCURF of type $(12 g)^{u}$ with partition $2^{4 g(u-1)} 3^{4 g(u-1) / 3}$ if and only if the following conditions are satisfied:

- $g \equiv 1,5 \bmod 6, u \equiv 1,4 \bmod 12$ and $u \geq 4$;

- $g \equiv 2,4 \bmod 6, u \equiv 1 \bmod 3, u \geq 4$ and $(g, u) \neq(2,4)$; 
- $g \equiv 3 \bmod 6, u \equiv 0,1 \bmod 4$ and $u \geq 4 ;$ and

- $g \equiv 0 \bmod 6, u \geq 4$ and $(g, u) \neq(6,4)$;

We note that this gives asymptotic existence for HCURFs with $g=12$ and $m=9$.

Similarly, since the existence of 4-frames is mostly known, CURGDDs may be used to obtain CURFs.

Lemma 4.12. [7] There exists a CURGDD of type $(3 n)^{4}$ with partition $2^{3 n} 3^{2 n}$ and $r=6 n$ resolution classes for all $n \geq 1$.

Theorem 4.13. If $u \geq 5, g \equiv 0 \bmod 3$ and $g(u-1) \equiv 0 \bmod 4$, there exists an HCURF of type $(3 g n)^{u}$ with partition $2^{3 n g(u-1) / 4} 3^{n g(u-1) / 2}$ for every $n>0$, with a small finite number of possible exceptions for each $g$.

Proof. Apply Theorem 3.2 taking a 4-frame as the master and the CURGDDs from Lemma 4.12 as ingredients. The existence of 4 -frames is known [12]; for a list of the possible exceptions we refer the reader to $[3,12]$ (see also [2]).

Next we give a construction based on breaking up an RBIBD in a systematic way.

Theorem 4.14. There exists an HCURF of type $(6 t+1)^{6 t+3}$ with partition $2^{6 t+1} 3^{2 t(6 t+1)}$ and $m=3 t+1$ for all $t \geq 0$.

Proof. We establish the existence of an $\mathrm{HCURF}_{6 t+1}$ of type $1^{6 t+3}$ and partition $2^{1} 3^{2 t}$ for all $t \geq 0$ that satisfy the conditions of Theorem 3.4. Start with a $\operatorname{RBIBD}(6 t+3,3,1)$ $(\operatorname{KTS}(6 t+3))$. For each resolution class of this design we will produce $6 t+3$ copies of it and modify them in a systematic way. For each block of the resolution class (there are $2 t+1$ blocks) we will modify three of the copies of the resolution class by replacing this block by the three resolution classes of a CURD with partition $1^{1} 2^{1}$. Doing this for each resolution class gives us $6 t+3$ resolution classes of the form $1^{1} 2^{1} 3^{2 t}$ which cover each edge from the original resolution class of the KTS exactly $6 t+1$ times. Each point appears as a block of size one the same number of times, independent of the choice of the point and so this is really an $\mathrm{HCURF}_{6 t+1}$ of type $1^{6 t+3}$ and partition $2^{1} 3^{2 t}$. Application of Theorem 3.4 gives the result.

\subsection{Prime Power Constructions}

We now present a number of constructions that use Theorem 3.4 to construct infinite families of CURFs.

Theorem 4.15. If $q$ is a prime power and there exists integers $d, k, m$ such that $d \leq$ $(q-1-k) / 2$ and $m k$ divides $d-(m k-k+1)$, then there exists a $H C U R F_{2(d+k-1) /(m k)}$ of type $(m k)^{q}$ with partition

$$
1^{m k(q-2 d-k-1)} 2^{d k+m\left(\begin{array}{c}
k \\
2
\end{array}\right)} k^{m}
$$

satisfying the conditions of Theorem 3.4. 
Proof. On $q$ points we make a class with one block of size $k$ and $d$ disjoint pairs. label each of the points uniquely but arbitrarily from the set $\{1,2, \ldots, k\}$. Label one of the pairs 0 , one of the pairs $j$ for each $j \in\{0,1, \ldots, m k-1\} \backslash\{1,2, \ldots, k-1\}$ and all of the remaining pairs from the set $\{0,1, \ldots, m k-1\}$ each label exactly $(d-(m k-k+1)) / m k$ times.

Develop this class through the group of affine functions on $\operatorname{GF}(q)$ (functions of the form $x \rightarrow a x+b$ where $a, b \in G F(q)$ and $a \neq 0)$. Blow up each point by $m k$. Lift the block of size $k$ to $m$ blocks of size $k$. The point labeled $j$ in the $i$ th such block will be lifted to the new point $i m+j$ where $0 \leq i<m$. If $\left\{(x, y),\left(x^{\prime}, y^{\prime}\right)\right\}$ is an edge contained in a block of size $k$ then add the pair $\left\{\left(x, y^{\prime}\right),\left(x^{\prime}, y\right)\right\}$. Now blow up each edge $\{x, y\}$ to a set of disjoint edges resolving groups $x$ and $y:\{(x, l),(y, l+m)\}$ where $l \in \mathbb{Z}_{m k}, m$ is the label on that edge and addition is mod $m k$.

Applying Theorem 3.4 we get:

Corollary 4.16. If $q$ is a prime power and there exists integers $d, k, m$ such that $d \leq$ $(q-1-k) / 2$ and $m k$ divides $d-(m k-k+1)$, then there exists a HCURF of type $(2(d+k-1))^{q}$ with partition

$$
1^{2(d+k-1)(q-2 d-k-1)} 2^{2(d+k-1)\left(d k+m\left(\begin{array}{c}
k \\
2
\end{array}\right)\right) / m k} k^{2(d+k-1) / k} .
$$

Using difference sets gives the following.

Theorem 4.17. If $q=2^{n}$ and there exists an (H)CURF of type $1^{q+1}$ with partition $\prod k^{p_{k}}$ and $r$ resolution classes then there exists an $(H) C U R F$ of type

$$
\left(\frac{r\left(q^{2}-1\right)}{\operatorname{gcd}\left(r, q^{2}-1\right)}\left(\frac{q^{2}-q}{q^{2}-1}+\frac{1}{r}\right)\right)^{q^{2}+q+1}
$$

with partition

$$
2^{\left(\frac{r\left(q^{2}-1\right)}{\operatorname{gcd}\left(r, q^{2}-1\right)}\left(\frac{q^{2}-q}{q^{2}-1}+\frac{1}{r}\right)\right) q^{2} / 2} \prod k^{\left(\frac{r\left(q^{2}-1\right)}{\operatorname{gcd}\left(r, q^{2}-1\right)}\left(\frac{q^{2}-q}{q^{2}-1}+\frac{1}{r}\right)\right) p_{k}}
$$

Proof. There exists a $\left(q^{2}+q+1, q+1,1\right)$ difference set, let $B$ be the base block. We note that $B^{c}$ is a $\left(q^{2}+q+1, q^{2}, q^{2}-q\right)$ difference set. Place $\left(q^{2}-1\right) / \operatorname{gcd}\left(r, q^{2}-1\right)$ copies of the (H)CURF on the points of $B$. Place $r / \operatorname{gcd}\left(r, q^{2}-1\right)$ copies of any 1-factorization on $B^{c}$. This gives $r\left(q^{2}-1\right) / \operatorname{gcd}\left(r, q^{2}-1\right)$ resolution classes. Develop these classes in $\mathbb{Z}_{q^{2}+q+1}$ to get a $(\mathrm{H}) \mathrm{CURF}_{\lambda}$ on $q^{2}+q+1$ points with partition $2^{q^{2} / 2} \prod k^{p_{k}}$ which satisfies the conditions of Theorem 3.4, where

$$
\lambda=\frac{r\left(q^{2}-1\right)}{\operatorname{gcd}\left(r, q^{2}-1\right)}\left(\frac{q^{2}-q}{q^{2}-1}+\frac{1}{r}\right) .
$$

The result follows from Theorem 3.4. 
Theorem 4.18. If $q$ is an odd prime power and there exists a CURD on $q+1$ points with partition $\prod k^{p_{k}}$ and $r$ resolution classes, then there exists a HCURF of type

$$
\left(\frac{r q^{2}}{\operatorname{gcd}\left(r, q^{2}\right)}\left(\frac{q^{2}-q}{q^{2}}+\frac{1}{r}\right)\right)^{q^{2}+q+1}
$$

with partition

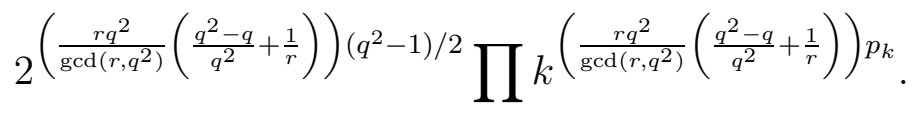

Proof. There exists a $\left(q^{2}+q+1, q+1,1\right)$ difference set, let $B$ be the base block. We note that $B^{c}$ is a $\left(q^{2}+q+1, q^{2}, q^{2}-q\right)$ difference set. Place $q^{2} / \operatorname{gcd}\left(r, q^{2}\right)$ copies of the CURD on the points of $B$. Place $r / \operatorname{gcd}\left(r, q^{2}\right)$ copies of any near 1 -factorization on $B^{c}$. This gives $r q^{2} / \operatorname{gcd}\left(r, q^{2}\right)$ partial resolution classes. Develop these classes in $\mathbb{Z}_{q^{2}+q+1}$ to get a (H) $\mathrm{CURF}_{\lambda}$ on $q^{2}+q+1$ points with partition $2^{q^{2} / 2} \prod k^{p_{k}}$ which satisfies the conditions of Theorem 3.4, where

$$
\lambda=\frac{r q^{2}}{\operatorname{gcd}\left(r, q^{2}\right)}\left(\frac{q-1}{q}+\frac{1}{r}\right) .
$$

The result follows from Theorem 3.4.

By using a $\operatorname{CURD}(q+1,(q+1) / 2,1,(q-1) / 3)\left(3\right.$-frames of type $\left.2^{(q+1) / 2}\right)$ where $q \equiv 1 \bmod 6$ and is a prime power as ingredients in Theorem 4.18 we have

Corollary 4.19. For all $q \equiv 1 \bmod 6$ a prime power there exists an HCURF of type $\left(\frac{q\left(q^{2}+2 q-1\right)}{2}\right)^{q^{2}+q+1}$ with partition

$$
2^{q\left(q^{2}+2 q-1\right)\left(q^{2}+1\right) / 4} 3^{q\left(q^{2}+2 q-1\right)(q-1) / 6} .
$$

Using Theorem 3.2 with CURGDDs produced by Corollary 4.1 and Theorem 4.2 from [7] (with three or four groups) as ingredients and frames with block sizes three or four [2] as the master, we get the following theorems.

Corollary 4.20. There exists an HCURF of type $(h q(q-m))^{u}$ with partition

$$
2^{h m(u-1)(q-m)} 3^{h(u-1)(q-m)(q-2 m) / 3}
$$

for all $q \geq 3$ a prime power, $0 \leq m \leq q / 2, u \geq 4, h \equiv 0 \bmod 2$ and $h(u-1) \equiv 0 \bmod 3$.

Corollary 4.21. When a 4-frame of type $h^{u}$ exists, $q \geq 3$ a prime power, $0 \leq m \leq q / 2$ and for all $g \equiv 0 \bmod 6$ then there exists an HCURF of type $(h g q(q-m))^{u}$ with partition $2^{h m g(u-1)(q-m)} 3^{h g(u-1)(q-m)(q-2 m) / 3}$

Corollary 4.22. When a 4-frame of type $h^{u}$ exists, then there exists an HCURF of type $(3 h)^{u}$ and partition $2^{3 h(u-1) / 4} 3^{h(u-1) / 2}$. 


\section{Conclusion}

Theorem 3.4 is a frame formulation of Theorem 3.3 from [7]. It provides a construction that can produce CURFs of index 1 from CURFs with higher index. We believe that this kind of construction is extremely potent and although it arose in the study of CURDs we anticipate that it will be a powerful tool for the construction of many more types of designs.

In this paper we have mainly constructed homogeneous CURFs, though many of the constructions given apply to the general case. The existence of non-homogeneous CURFs with constant group size is surprising, as are the weak combinatorial constraints that sometimes force homogeneity as in the proof of Theorem 2.2. The consideration of nonhomogeneous CURFs is an open problem. In particular, non-homogeneity may provide avenues for new future constructions, even those that produce homogeneous objects.

Frame constructions often gain in power by the consideration of multiple group sizes. Frames with multiple group sizes are forced to have multiple partitions, at least one for each group size, thus strictly speaking these are not class-uniform structures. However, such designs may be very useful for finding class-uniform objects. The existence of multiple partitions is reminiscent of the fact that resolvable designs with holes have two types of resolution classes. The consideration of multiple partitions in a class-uniform context remains an interesting open problem.

\section{A Appendix}

We present some of the HCURFs required for the results in Section 4.1. Generally, these are cyclic designs which satisfy the conditions of Theorem 3.4. In some cases the triples are common to all classes and we then give them separately at the top. For other cases the triples are given with the class.

\section{A.1 HCURFs of type $4^{9 t+1}$}

We present the family of $\mathrm{CURF}_{4}$ of type $1^{9 t+1}$ with partition $2^{3 t} 3^{t}$ for $t=1$ to 5 . The cases $t=1$ to 100 may be found in [5].

$\mathrm{A} \mathrm{CURF}_{4}$ of type $1^{10}$ with partition $2^{3} 3^{1}(\mathrm{t}=1)$

$$
\begin{aligned}
& \text { Triples }\{1,2,4\} \\
& \begin{array}{llll}
8: & \{0,5\} & \{6,7\} & \{9,3\} \\
9: & \{0,5\} & \{6,8\} & \{3,7\} \\
9: & \{3,7\} & \{5,8\} & \{6,0\}
\end{array}
\end{aligned}
$$

$\mathrm{A} \mathrm{CURF}_{4}$ of type $1^{19}$ with partition $2^{6} 3^{2}(\mathrm{t}=2)$

$$
\begin{array}{rllllll}
\multicolumn{8}{c}{\text { Triples }} & \{0,1,3\} & \{2,6,11\} & \\
10: & \{9,17\} & \{12,13\} & \{4,15\} & \{18,5\} & \{7,14\} & \{8,16\} \\
17: & \{9,4\} & \{16,7\} & \{5,13\} & \{14,10\} & \{12,18\} & \{8,15\} \\
18: & \{8,5\} & \{4,16\} & \{7,13\} & \{9,15\} & \{10,17\} & \{12,14\}
\end{array}
$$


A CURF $_{4}$ of type $1^{28}$ with partition $2^{9} 3^{3}(\mathrm{t}=3)$

$$
\text { Triples }\{1,2,4\} \quad\{3,7,12\} \quad\{5,11,18\}
$$

$\begin{array}{llllllllll}25: & \{0,14\} & \{8,20\} & \{23,27\} & \{10,19\} & \{15,26\} & \{13,24\} & \{22,6\} & \{9,17\} & \{16,21\} \\ 20: & \{0,14\} & \{23,25\} & \{13,21\} & \{17,6\} & \{9,19\} & \{16,26\} & \{22,10\} & \{24,27\} & \{8,15\} \\ 24: & \{14,20\} & \{27,9\} & \{0,13\} & \{6,16\} & \{8,19\} & \{21,22\} & \{26,10\} & \{17,25\} & \{15,23\}\end{array}$

A CURF $_{4}$ of type $1^{37}$ with partition $2^{12} 3^{4}(\mathrm{t}=4)$

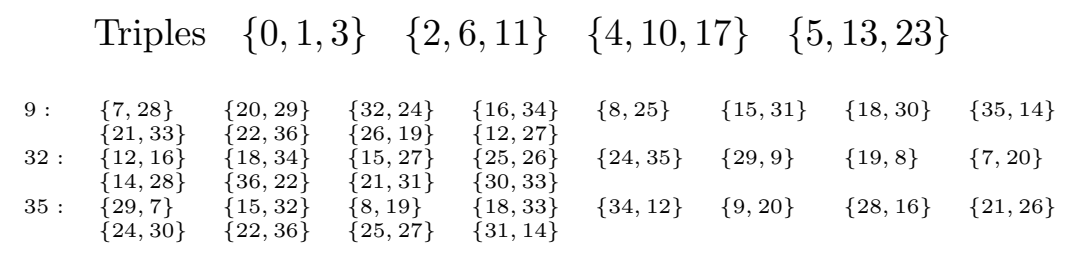

$\mathrm{A} \mathrm{CURF}_{4}$ of type $1^{46}$ with partition $2^{15} 3^{5}(\mathrm{t}=5)$

$$
\text { Triples }\{1,2,4\} \quad\{3,7,12\} \quad\{5,11,18\} \quad\{6,14,24\} \quad\{8,19,33\}
$$

$\begin{array}{lllllllll}37: & \{0,23\} & \{20,22\} & \{31,36\} & \{34,44\} & \{35,9\} & \{21,43\} & \{27,39\} & \{13,41\} \\ & \{28,42\} & \{17,25\} & \{45,29\} & \{40,16\} & \{26,38\} & \{10,32\} & \{15,30\} & \\ 44: & \{0,23\} & \{13,40\} & \{26,10\} & \{27,30\} & \{21,37\} & \{29,36\} & \{20,42\} & \{17,32\} \\ & \{22,39\} & \{16,31\} & \{38,9\} & \{35,41\} & \{34,15\} & \{28,43\} & \{25,45\} & \\ 21: & \{28,32\} & \{36,27\} & \{22,35\} & \{20,41\} & \{37,38\} & \{23,40\} & \{9,26\} & \{45,10\} \\ & \{25,44\} & \{39,13\} & \{42,16\} & \{29,17\} & \{30,0\} & \{31,43\} & \{15,34\} & \end{array}$

\section{A.2 HCURFs of type $5^{18 t+1}$}

A CURF 5 of type $1^{19}$ with partition $2^{3} 3^{4}(t=1)$ satisfying $\lambda$ blowup. Point set $\mathbb{Z}_{19}$. Develop the following 3 partial classes, mod 19

$\begin{array}{lllllll}\{0,1,4\} & \{2,3,6\} & \{7,8,11\} & \{9,10,13\} & \{5,15\} & \{12,17\} & \{14,18\} \\ \{0,2,9\} & \{1,3,10\} & \{4,6,13\} & \{5,7,14\} & \{12,15\} & \{11,17\} & \{8,16\} \\ \{0,5,11\} & \{1,6,12\} & \{2,7,13\} & \{3,8,14\} & \{4,16\} & \{15,17\} & \{9,10\}\end{array}$

A CURF 5 of type $1^{37}$ with partition $2^{6} 3^{8}(\mathrm{t}=2)$ satisfying $\lambda$ blowup. Point set $\mathbb{Z}_{37}$. Develop the following 3 partial classes, mod 37

$\begin{array}{lllllll}\{0,1,3\} & \{4,5,7\} & \{8,9,11\} & \{12,13,15\} & \{2,6,28\} & \{10,14,36\} & \{27,31,16\} \\ \{29,33,18\} & \{17,20\} & \{21,25\} & \{34,35\} & \{19,24\} & \{23,30\} & \{22,32\} \\ & & & & & & \\ \{0,5,14\} & \{1,6,15\} & \{2,7,16\} & \{3,8,17\} & \{4,10,29\} & \{21,27,9\} & \{24,30,12\} \\ \{25,11,13\} & \{33,11\} & \{22,28\} & \{18,32\} & \{23,34\} & \{35,26\} & \{20,36\} \\ \{0,7,17\} & \{1,8,18\} & \{2,9,19\} & \{3,10,20\} & \{4,12,25\} & \{5,13,26\} & \{6,14,27\} \\ \{15,23,36\} & \{34,16\} & \{11,28\} & \{29,31\} & \{24,32\} & \{22,35\} & \{21,33\}\end{array}$

A CURF 5 of type $1^{55}$ with partition $2^{9} 3^{12}(\mathrm{t}=3)$ satisfying $\lambda$ blowup. Point set $\mathbb{Z}_{55}$. Develop the following 3 partial classes, mod 55

$\begin{array}{lllllll}\{0,1,3\} & \{4,5,7\} & \{8,9,11\} & \{12,13,15\} & \{10,14,19\} & \{16,20,25\} & \{17,21,26\} \\ \{18,22,27\} & \{23,29,39\} & \{24,30,40\} & \{28,34,44\} & \{31,37,47\} & \{53,50\} & \{52,38\} \\ \{6,32\} & \{2,36\} & \{33,41\} & \{45,46\} & \{42,54\} & \{49,51\} & \{35,48\} \\ & & & & & & \\ \{0,7,32\} & \{1,8,33\} & \{2,9,34\} & \{3,10,35\} & \{11,19,40\} & \{12,20,41\} & \{13,21,42\} \\ \{14,22,43\} & \{4,15,46\} & \{5,16,47\} & \{6,17,48\} & \{38,49,25\} & \{37,44\} & \{24,29\} \\ \{39,30\} & \{18,53\} & \{51,26\} & \{27,50\} & \{52,28\} & \{36,54\} & \{45,23\} \\ & & & & & \\ \{0,12,27\} & \{1,13,28\} & \{2,14,29\} & \{3,15,30\} & \{4,18,40\} & \{5,19,41\} & \{6,20,42\} \\ \{7,21,43\} & \{8,25,45\} & \{9,26,46\} & \{16,33,53\} & \{17,34,54\} & \{50,23\} & \{22,11\} \\ \{36,52\} & \{44,38\} & \{10,48\} & \{32,51\} & \{35,31\} & \{47,37\} & \{24,39\}\end{array}$




\section{A.3 HCURFs of type $5^{24 t+1}$}

$\mathrm{A} \mathrm{CURF}_{5}$ of type $1^{25}$ with partition $2^{9} 3^{2}(\mathrm{t}=1)$

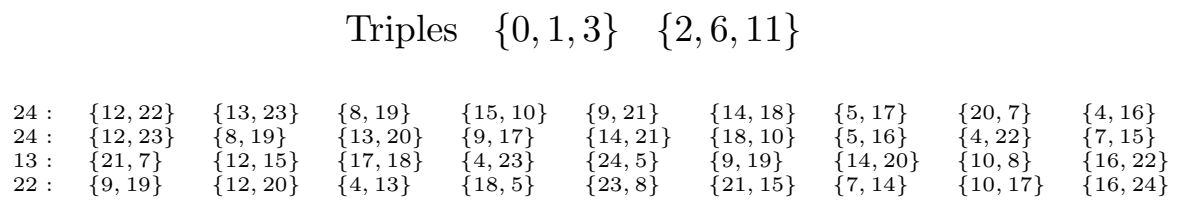

$\mathrm{A} \mathrm{CURF}_{5}$ of type $1^{49}$ with partition $2^{18} 3^{4}(\mathrm{t}=2)$

Triples $\{0,1,3\} \quad\{2,6,11\}$

$\{4,10,17\}$

$\{5,13,23\}$

$\begin{array}{rllllllll}46: & \{18,25\} & \{47,12\} & \{33,7\} & \{29,44\} & \{35,38\} & \{40,15\} & \{16,30\} & \{9,21\} \\ & \{8,28\} & \{32,43\} & \{24,41\} & \{14,37\} & \{20,39\} & \{22,42\} & \{26,34\} & \{27,31\} \\ & \{45,19\} & \{36,48\} & & & & & & \\ 44: & \{40,15\} & \{7,24\} & \{31,41\} & \{14,29\} & \{28,42\} & \{36,9\} & \{45,20\} & \{35,8\} \\ & \{27,46\} & \{26,43\} & \{30,47\} & \{25,39\} & \{16,32\} & \{22,33\} & \{18,38\} & \{21,19\} \\ & \{37,12\} & \{34,48\} & & & & & & \\ 7: & \{21,45\} & \{40,12\} & \{14,35\} & \{26,37\} & \{42,15\} & \{48,27\} & \{28,34\} & \{8,20\} \\ & \{38,29\} & \{31,47\} & \{19,39\} & \{18,36\} & \{43,44\} & \{22,33\} & \{41,25\} & \{30,46\} \\ 32: & \{24,9\} & \{16,32\} & & & & & & \\ & \{20,40\} & \{14,35\} & \{24,43\} & \{16,37\} & \{18,30\} & \{28,47\} & \{26,41\} & \{7,39\} \\ & \{38,12\} & \{48,22\} & \{8,44\} & \{46,19\} & \{9,31\} & \{29,34\} & \{21,33\} & \{45,15\} \\ & \{27,42\} & \{25,36\} & & & & & & \end{array}$

\section{A.4 HCURFs of type $6^{2 t+1}$}

We give an HCURF of type $6^{2 t+1}$ for $t=3,4,5,6$, corresponding to $u=7,9,11,13, t=2$ $(u=5)$ is given in the text. This is a generating set for $u \equiv 1 \bmod 2[1]$, with the 20 possible exceptions:

$$
u \in\{43,51,59,71,75,83,87,95,107,111,115,119,131,135,139,167,179,183,191,195\} .
$$

A CURF of type $6^{7}$ with partition $2^{9} 3^{6}(\mathrm{t}=3)$ Point set $\mathbb{Z}_{6} \times \mathbb{Z}_{7}$, groups $\mathbb{Z}_{6} \times\{i\}, i \in \mathbb{Z}_{7}$. Develop the following 4 classes, each missing group $\mathbb{Z}_{6} \times\{0\}, \bmod (-, 7)$

$\begin{array}{llll}\{(2,5),(0,4),(1,6)\} & \{(1,1),(5,3),(4,6)\} & \{(3,3),(2,2),(0,6)\} & \{(2,1),(1,3),(3,6)\} \\ \{(5,6),(3,2),(2,3)\} & \{(4,2),(0,1),(5,4)\} & \{(4,5),(4,4)\} & \{(4,3),(3,4)\} \\ \{(0,2),(4,1)\} & \{(2,4),(2,6)\} & \{(0,3),(0,5)\} & \{(1,2),(1,4)\} \\ \{(1,5),(5,2)\} & \{(5,1),(5,5)\} & \{(3,5),(3,1)\} & \\ & & & \\ & & & \\ \{(3,3),(1,4),(5,5)\} & \{(4,4),(0,2),(1,1)\} & \{(1,5),(5,3),(4,2)\} & \{(1,6),(2,2),(0,3)\} \\ \{(3,1),(5,6),(1,3)\} & \{(5,1),(0,6),(2,3)\} & \{(2,4),(1,2)\} & \{(0,4),(0,5)\} \\ \{(5,4),(5,2)\} & \{(4,3),(4,6)\} & \{(2,6),(3,2)\} & \{(2,5),(2,1)\} \\ \{(0,1),(4,5)\} & \{(3,5),(3,6)\} & \{(3,4),(4,1)\} & \\ \{(4,5),(5,3),(2,6)\} & \{(2,5),(4,2),(1,1)\} & \{(5,2),(3,6),(0,3)\} & \{(4,6),(3,1),(1,4)\} \\ \{(1,3),(0,2),(2,4)\} & \{(0,6),(5,4),(3,5)\} & \{(5,1),(1,2)\} & \{(3,2),(3,4)\} \\ \{(0,4),(2,2)\} & \{(1,6),(0,1)\} & \{(5,5),(2,3)\} & \{(2,1),(3,3)\} \\ \{(1,5),(4,4)\} & \{(0,5),(5,6)\} & \{(4,1),(4,3)\} & \\ & & & \\ \{(3,2),(1,1),(0,4)\} & \{(3,3),(0,1),(4,6)\} & \{(2,1),(5,2),(4,3)\} & \{(2,4),(3,1),(4,2)\} \\ \{(4,5),(5,1),(2,2)\} & \{(3,6),(4,1),(0,5)\} & \{(0,3),(0,6)\} & \{(0,2),(5,6)\} \\ \{(1,5),(1,4)\} & \{(4,4),(2,3)\} & \{(5,3),(5,4)\} & \{(1,3),(3,5)\} \\ \{(3,4),(5,5)\} & \{(1,6),(1,2)\} & \{(2,5),(2,6)\} & \end{array}$

A CURF of type $6^{9}$ with partition $2^{12} 3^{8}(\mathrm{t}=4)$ Point set $\mathbb{Z}_{6} \times \mathbb{Z}_{9}$, groups $\mathbb{Z}_{6} \times\{i\}, i \in \mathbb{Z}_{9}$. Develop the following 4 classes, each missing group $\mathbb{Z}_{6} \times\{0\}, \bmod (-, 9)$

$\begin{array}{llll}\{(5,7),(2,1),(4,2)\} & \{(2,5),(4,3),(0,1)\} & \{(1,4),(0,3),(4,6)\} & \{(5,5),(1,1),(2,7)\} \\ \{(0,8),(3,3),(4,4)\} & \{(1,3),(0,4),(3,7)\} & \{(3,1),(5,3),(2,2)\} & \{(2,4),(5,8),(1,5)\} \\ \{(4,1),(3,5)\} & \{(0,2),(0,5)\} & \{(1,8),(0,6)\} & \{(5,6),(5,1)\} \\ \{(4,7),(1,2)\} & \{(0,7),(2,8)\} & \{(3,6),(3,8)\} & \{(5,4),(5,2)\} \\ \{(3,2),(4,8)\} & \{(4,5),(2,3)\} & \{(1,6),(1,7)\} & \{(3,4),(2,6)\}\end{array}$




$\begin{array}{llll}\{(4,8),(1,4),(2,5)\} & \{(0,1),(1,7),(5,4)\} & \{(2,1),(3,2),(4,6)\} & \{(5,8),(1,1),(0,6)\} \\ \{(0,2),(2,4),(4,1)\} & \{(5,6),(4,3),(0,5)\} & \{(0,7),(3,8),(5,5)\} & \{(4,2),(5,1),(3,3)\} \\ \{(0,4),(1,2)\} & \{(3,1),(0,3)\} & \{(3,4),(2,2)\} & \{(5,3),(5,2)\} \\ \{(2,8),(2,7)\} & \{(5,7),(1,6)\} & \{(2,3),(2,6)\} & \{(3,7),(3,6)\} \\ \{(3,5),(0,8)\} & \{(4,7),(4,5)\} & \{(1,3),(4,4)\} & \{(1,5),(1,8)\} \\ & & & \\ \{(1,1),(0,7),(4,8)\} & \{(2,2),(1,7),(3,6)\} & \{(3,7),(5,1),(0,5)\} & \{(4,7),(2,3),(5,8)\} \\ \{(3,4),(5,5),(1,6)\} & \{(5,7),(4,3),(0,8)\} & \{(3,3),(0,4),(2,7)\} & \{(1,2),(4,1),(3,8)\} \\ \{(0,6),(5,3)\} & \{(5,6),(3,1)\} & \{(0,3),(2,8)\} & \{(3,5),(3,2)\} \\ \{(2,1),(2,5)\} & \{(1,8),(1,4)\} & \{(4,2),(5,4)\} & \{(0,2),(0,1)\} \\ \{(1,5),(1,3)\} & \{(4,6),(4,5)\} & \{(5,2),(4,4)\} & \{(2,6),(2,4)\} \\ & & & \\ \{(4,7),(2,8),(0,1)\} & \{(3,8),(4,6),(5,3)\} & \{(3,3),(5,2),(0,7)\} & \{(1,4),(3,7),(4,1)\} \\ \{(1,5),(3,6),(2,3)\} & \{(2,1),(0,2),(1,7)\} & \{(1,2),(3,4),(2,7)\} & \{(2,5),(5,8),(1,3)\} \\ \{(1,1),(4,4)\} & \{(1,8),(5,1)\} & \{(2,6),(5,5)\} & \{(3,1),(3,5)\} \\ \{(0,4),(0,6)\} & \{(5,4),(5,7)\} & \{(0,5),(2,2)\} & \{(5,6),(2,4)\} \\ \{(0,8),(0,3)\} & \{(4,3),(4,8)\} & \{(3,2),(1,6)\} & \{(4,5),(4,2)\}\end{array}$

A CURF of type $6^{11}$ with partition $2^{15} 3^{10}(\mathrm{t}=5)$ Point set $\mathbb{Z}_{6} \times \mathbb{Z}_{11}$, groups $\mathbb{Z}_{6} \times\{i\}, i \in$ $\mathbb{Z}_{11}$. Develop the following 4 classes, each missing group $\mathbb{Z}_{6} \times\{0\}, \bmod (-, 11)$

$\begin{array}{llll}\{(1,8),(0,1),(4,4)\} & \{(1,1),(3,2),(0,3)\} & \{(1,4),(1,9),(2,10)\} & \{(5,7),(4,8),(5,6)\} \\ \{(3,10),(2,5),(2,4)\} & \{(5,4),(4,7),(0,9)\} & \{(5,1),(2,8),(3,7)\} & \{(0,8),(3,5),(3,4)\} \\ \{(3,1),(3,8),(1,6)\} & \{(5,8),(2,1),(1,10)\} & \{(5,3),(0,7)\} & \{(3,3),(0,2)\} \\ \{(2,6),(4,9)\} & \{(4,10),(5,5)\} & \{(1,3),(5,9)\} & \{(2,2),(1,5)\} \\ \{(3,6),(4,5)\} & \{(0,10),(4,6)\} & \{(5,2),(0,5)\} & \{(2,3),(2,9)\} \\ \{(0,4),(4,3)\} & \{(0,6),(3,9)\} & \{(2,7),(5,10)\} & \{(4,2),(1,7)\} \\ \{(4,1),(1,2)\} & & & \\ & & & \\ \{(1,4),(1,7),(0,10)\} & \{(1,2),(0,7),(1,6)\} & \{(5,7),(3,4),(2,2)\} & \{(1,9),(4,7),(3,1)\} \\ \{(5,3),(0,1),(0,5)\} & \{(5,8),(4,6),(2,10)\} & \{(4,2),(4,9),(3,6)\} & \{(5,9),(5,6),(1,10)\} \\ \{(2,7),(0,4),(2,9)\} & \{(4,1),(4,4),(3,10)\} & \{(4,3),(0,2)\} & \{(5,5),(5,10)\} \\ \{(1,5),(5,2)\} & \{(2,3),(3,7)\} & \{(0,9),(2,5)\} & \{(5,4),(3,2)\} \\ \{(3,3),(3,5)\} & \{(4,10),(0,8)\} & \{(3,8),(2,1)\} & \{(0,6),(2,8)\} \\ \{(5,1),(2,4)\} & \{(1,8),(2,6)\} & \{(1,3),(4,5)\} & \{(4,8),(0,3)\} \\ \{(3,9),(1,1)\} & & & \\ & & & \\ \{(4,2),(1,8),(1,9)\} & \{(5,4),(5,2),(4,1)\} & \{(4,10),(3,9),(3,6)\} & \{(0,3),(0,4),(1,7)\} \\ \{(4,5),(4,4),(2,3)\} & \{(0,1),(0,7),(2,5)\} & \{(2,4),(1,1),(5,6)\} & \{(5,8),(3,10),(2,7)\} \\ \{(2,1),(5,7),(1,5)\} & \{(1,4),(2,9),(4,7)\} & \{(4,6),(0,2)\} & \{(4,9),(2,10)\} \\ \{(3,2),(1,6)\} & \{(0,9),(1,10)\} & \{(0,6),(5,10)\} & \{(0,8),(0,5)\} \\ \{(5,9),(3,1)\} & \{(2,6),(3,4)\} & \{(5,1),(5,5)\} & \{(1,3),(3,7)\} \\ \{(3,5),(2,8)\} & \{(3,3),(0,10)\} & \{(4,8),(2,2)\} & \{(1,2),(4,3)\} \\ \{(3,8),(5,3)\} & & & \\ & & & \\ \{(4,8),(5,2),(2,3)\} & \{(5,8),(0,9),(3,7)\} & \{(4,5),(4,7),(0,10)\} & \{(0,3),(2,2),(0,5)\} \\ \{(3,4),(3,9),(1,10)\} & \{(4,9),(5,5),(3,1)\} & \{(0,7),(2,8),(1,9)\} & \{(2,6),(4,3),(2,10)\} \\ \{(3,8),(0,2),(5,7)\} & \{(0,8),(5,9),(3,2)\} & \{(5,3),(1,2)\} & \{(1,5),(4,2)\} \\ \{(4,10),(4,4)\} & \{(2,4),(2,7)\} & \{(4,6),(5,10)\} & \{(4,1),(3,3)\} \\ \{(1,1),(2,5)\} & \{(0,1),(5,4)\} & \{(5,1),(1,8)\} & \{(2,9),(3,10)\} \\ \{(1,3),(5,6)\} & \{(1,7),(3,5)\} & \{(2,1),(0,6)\} & \{(0,4),(3,6)\} \\ \{(1,4),(1,6)\} & & & \end{array}$

A CURF of type $6^{13}$ with partition $2^{18} 3^{12}(\mathrm{t}=6)$ Point set $\mathbb{Z}_{6} \times \mathbb{Z}_{13}$, groups $\mathbb{Z}_{6} \times\{i\}, i \in$ $\mathbb{Z}_{13}$. Develop the following 4 classes, each missing group $\mathbb{Z}_{6} \times\{0\}, \bmod (-, 13)$

$\begin{array}{llll}\{(1,5),(4,4),(5,10)\} & \{(3,5),(3,9),(2,11)\} & \{(4,7),(0,1),(0,2)\} & \{(4,9),(3,4),(3,7)\} \\ \{(4,11),(2,8),(3,12)\} & \{(5,2),(4,6),(0,5)\} & \{(5,7),(1,12),(1,1)\} & \{(1,8),(4,1),(4,12)\} \\ \{(2,10),(5,4),(0,6)\} & \{(3,3),(1,1),(3), 10)\} & \{(2,2),(1,9),(2,1)\} & \{(2,5),(0,7),(5,6)\} \\ \{(1,4),(3,11)\} & \{(1,3),(3,1)\} & \{(0,10),(4,5)\} & \{(0,8),(1,10)\} \\ \{(0,4),(2,7)\} & \{(2,3),(5,5)\} & \{(4,2),(5,3)\} & \{(2,4),(1,6)\} \\ \{(3,2),(0,9)\} & \{(4,8),(5,12)\} & \{(5,8),(2,9)\} & \{(0,11),(5,1)\} \\ \{(0,3),(5,9)\} & \{(5,11),(3,6)\} & \{(3,8),(1,7)\} & \{(2,6),(2,12)\} \\ \{(4,10),(1,2)\} & \{(4,3),(0,12)\} & & \\ & & & \\ \{(0,4),(4,3),(1,11)\} & \{(4,4),(4,12),(5,9)\} & \{(1,2),(1,7),(3,11)\} & \{(3,2),(5,1),(3,4)\} \\ \{(2,7),(2,1)),(3,12)\} & \{(2,1),(2,4),(1,5)\} & \{(5,4),(5,11),(5,2)\} & \{(3,5),(5,3),(0,2)\} \\ \{(3,10),(4,5),(4,11)\} & \{(2,10),(2,8),(5,5)\} & \{(1,9),(1,10),(0,6)\} & \{(0,1),(3,8),(5,10)\} \\ \{(0,8),(3,3)\} & \{(2,6),(4,2)\} & \{(1,1),(2,3)\} & \{(3,9),(1,12)\} \\ \{(0,10),(2,2)\} & \{(2,5),(0,3)\} & \{(5,6),(4,8)\} & \{(0,5),(5,12)\} \\ \{(3,1),(4,7)\} & \{(0,12),(4,1)\} & \{(4,10),(1,3)\} & \{(0,11),(1,8)\} \\ \{(2,12),(3,7)\} & \{(1,4),(5,7)\} & \{(3,6),(0,7)\} & \{(4,6),(0,9)\} \\ \{(1,6),(2,9)\} & \{(5,8),(4,9)\} & & \\ & & & \\ \{(5,5),(0,1),(2,2)\} & \{(2,12),(3,8),(4,4)\} & \{(5,1),(1,12),(1,2)\} & \{(4,7),(1,9),(2,10)\} \\ \{(1,1),(0,5),(0,8)\} & \{(5,4),(4,9),(2,11)\} & \{(2,1),(0,7),(3,3)\} & \{(1,8),(4,10),(0,3)\} \\ \{(2,7),(4,8),(0,12)\} & \{(3,10),(1,4),(0,6)\} & \{(5,7),(5,2),(1,6)\} & \{(3,1),(5,9),(5,10)\} \\ \{(4,2),(2,9)\} & \{(1,11),(4,1)\} & \{(1,10),(2,4)\} & \{(3,11),(1,3)\} \\ \{(0,10),(3,12)\} & \{(5,3),(0,11)\} & \{(0,2),(2,8)\} & \{(5,11),(3,5)\} \\ \{(2,6),(0,9)\} & \{(2,5),(3,4)\} & \{(3,2),(4,12)\} & \{(4,11),(2,3)\} \\ \{(4,6),(5,8)\} & \{(3,7),(3,6)\} & \{(5,12),(4,5)\} & \{(0,4),(1,5)\} \\ \{(5,6),(4,3)\} & \{(3,9),(1,7)\} & & \end{array}$




$\begin{array}{llll}\{(2,8),(1,11),(4,7)\} & \{(0,12),(0,6),(0,1)\} & \{(2,1),(4,5),(3,11)\} & \{(4,8),(2,6),(0,10)\} \\ \{(0,4),(0,8),(3,5)\} & \{(2,4),(3,10),(0,5)\} & \{(5,8),(3,4),(5,11)\} & \{(1,1),(0,2),(1,10)\} \\ \{(2,10),(2,5),(5,1)\} & \{(3,1),(4,12),(3,9)\} & \{(4,1),(4,11),(4,2)\} & \{(5,10),(1,6),(1,12)\} \\ \{(1,7),(4,4)\} & \{(3,6),(2,3)\} & \{(5,6),(0,11)\} & \{(5,7),(2,2)\} \\ \{(1,9),(5,3)\} & \{(5,9),(2,11)\} & \{(1,8),(4,9)\} & \{(3,3),(5,4)\} \\ \{(3,2),(4,6)\} & \{(2,9),(4,3)\} & \{(1,3),(2,7)\} & \{(3,12),(5,2)\} \\ \{(0,3),(5,5)\} & \{(1,5),(3,8)\} & \{(1,4),(2,12)\} & \{(3,7),(0,9)\} \\ \{(5,12),(1,2)\} & \{(0,7),(4,10)\} & & \end{array}$

\section{A.5 HCURFs of type $7^{8 t+1}$}

For $t \in\{2,3,4,5,6,7,8,11,12,13,14,15,16\}$ we give a $\mathrm{CURF}_{7}$ of type $1^{8 t+1}$ with partition $2^{t} 3^{2 t}$ satisfying $\lambda$ blow-up (Theorem 3.4), $t=1, u=9$ is given by Theorem 4.14. This corresponds to the generating set $u \in\{9,17,25,33,41,49,57,65,89,97,105,113,121,129\}$ from [2], leaving 66 possible exceptions. In each case we give 4 partial classes over the point set $\mathbb{Z}_{8 t+1}$, each missing one point. The design is obtained by developing these 4

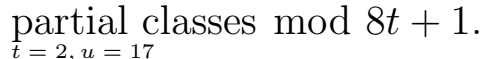

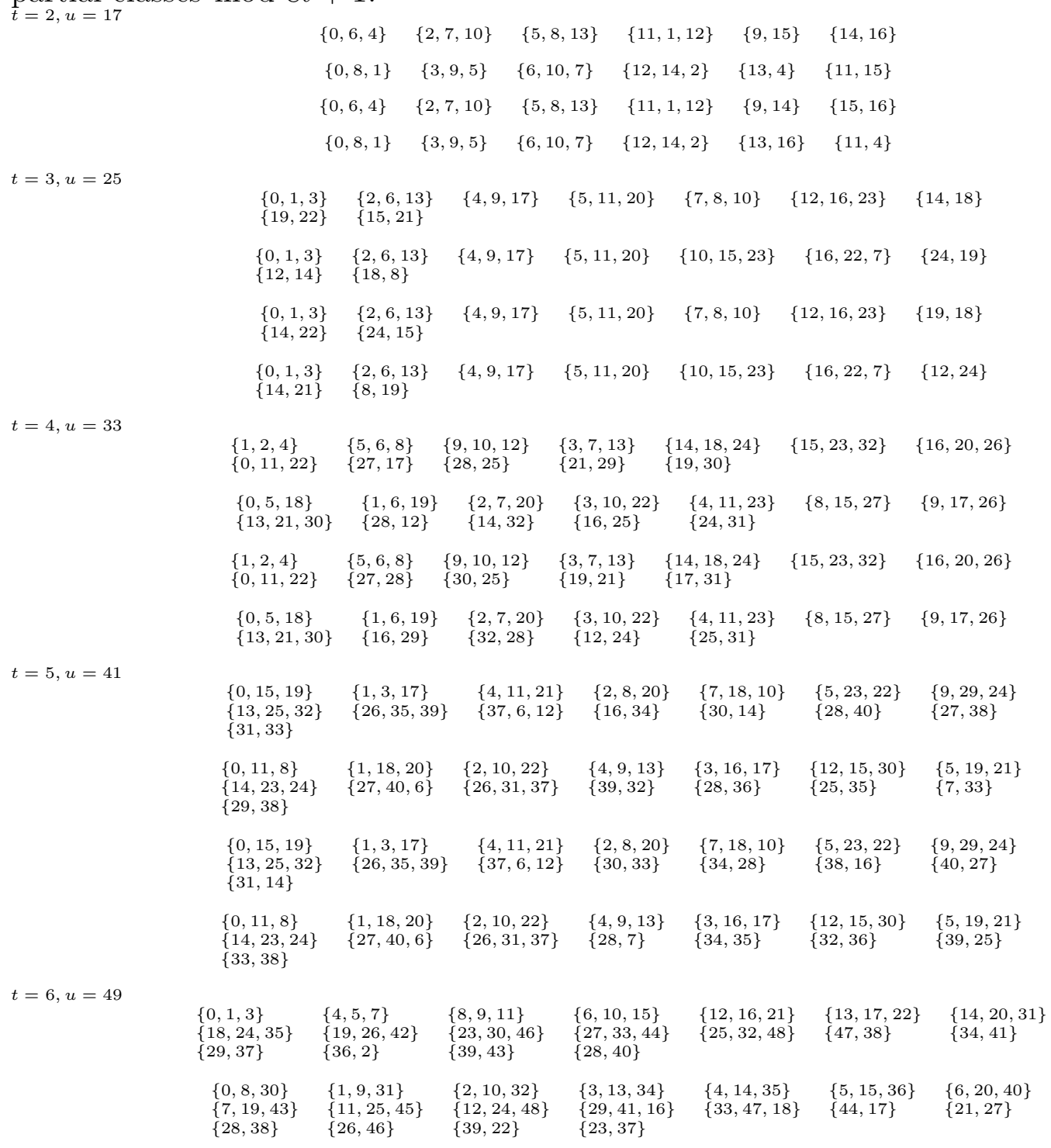




\begin{tabular}{|c|c|c|c|c|c|c|c|}
\hline & $\begin{array}{l}\{0,1,3\} \\
\{18,24,35\} \\
\{41,43\}\end{array}$ & $\begin{array}{l}\{4,5,7\} \\
\{19,26,42\} \\
\{37,38\}\end{array}$ & $\begin{array}{l}\{8,9,11\} \\
\{23,30,46\} \\
\{29,45\}\end{array}$ & $\begin{array}{l}\{6,10,15\} \\
\{27,33,44\} \\
\{2,28\}\end{array}$ & $\begin{array}{l}\{12,16,21\} \\
\{25,32,48\}\end{array}$ & $\begin{array}{l}\{13,17,22\} \\
\{36,39\}\end{array}$ & $\begin{array}{l}\{14,20,31\} \\
\{34,47\}\end{array}$ \\
\hline & $\begin{array}{l}\{0,8,30\} \\
\{7,19,43\} \\
\{46,21\}\end{array}$ & $\begin{array}{l}\{1,9,31\} \\
\{11,25,45\} \\
\{42,23\}\end{array}$ & $\begin{array}{l}\{2,10,32\} \\
\{12,24,48\} \\
\{28,39\}\end{array}$ & $\begin{array}{l}\{3,13,34\} \\
\{29,41,16\} \\
\{17,38\}\end{array}$ & $\begin{array}{l}\{4,14,35\} \\
\{33,47,18\}\end{array}$ & $\begin{array}{l}\{5,15,36\} \\
\{44,26\}\end{array}$ & $\begin{array}{l}\{6,20,40\} \\
\{22,27\}\end{array}$ \\
\hline$t=7, u=57$ & $\begin{array}{l}\{1,2,4\} \\
\{20,26,33\} \\
\{30,49\}\end{array}$ & $\begin{array}{l}\{5,6,8\} \\
\{24,32,50\} \\
\{39,54\}\end{array}$ & $\begin{array}{l}\{9,10,12\} \\
\{29,35,42\} \\
\{45,53\}\end{array}$ & $\begin{array}{l}\{7,11,16\} \\
\{44,56,27\} \\
\{40,46\}\end{array}$ & $\begin{array}{l}\{13,17,22\} \\
\{48,3,31\} \\
\{51,52\}\end{array}$ & $\begin{array}{l}\{14,18,23\} \\
\{41,55,25\} \\
\{36,43\}\end{array}$ & $\begin{array}{l}\{15,21,28\} \\
\{0,19,38\} \\
\{34,37\}\end{array}$ \\
\hline & $\begin{array}{l}\{0,8,26\} \\
\{10,21,42\} \\
\{31,53\}\end{array}$ & $\begin{array}{l}\{1,9,27\} \\
\{11,23,51\} \\
\{43,52\}\end{array}$ & $\begin{array}{l}\{2,12,35\} \\
\{5,20,40\} \\
\{33,44\}\end{array}$ & $\begin{array}{l}\{3,13,36\} \\
\{15,30,50\} \\
\{48,28\}\end{array}$ & $\begin{array}{l}\{4,14,37\} \\
\{32,46,16\} \\
\{45,22\}\end{array}$ & $\begin{array}{l}\{6,17,38\} \\
\{41,55,25\} \\
\{47,29\}\end{array}$ & $\begin{array}{l}\{7,18,39\} \\
\{19,34,54\} \\
\{24,49\}\end{array}$ \\
\hline & $\begin{array}{l}\{1,2,4\} \\
\{20,26,33\} \\
\{40,45\}\end{array}$ & $\begin{array}{l}\{5,6,8\} \\
\{24,32,50\} \\
\{37,49\}\end{array}$ & $\begin{array}{l}\{9,10,12\} \\
\{29,35,42\} \\
\{34,51\}\end{array}$ & $\begin{array}{l}\{7,11,16\} \\
\{44,56,27\} \\
\{36,52\}\end{array}$ & $\begin{array}{l}\{13,17,22\} \\
\{48,3,31\} \\
\{43,47\}\end{array}$ & $\begin{array}{l}\{14,18,23\} \\
\{41,55,25\} \\
\{53,39\}\end{array}$ & $\begin{array}{l}\{15,21,28\} \\
\{0,19,38\} \\
\{30,54\}\end{array}$ \\
\hline & $\begin{array}{l}\{0,8,26\} \\
\{10,21,42\} \\
\{53,22\}\end{array}$ & $\begin{array}{l}\{1,9,27\} \\
\{11,23,51\} \\
\{29,56\}\end{array}$ & $\begin{array}{l}\{2,12,35\} \\
\{5,20,40\} \\
\{45,47\}\end{array}$ & $\begin{array}{l}\{3,13,36\} \\
\{15,30,50\} \\
\{28,49\}\end{array}$ & $\begin{array}{l}\{4,14,37\} \\
\{32,46,16\} \\
\{43,33\}\end{array}$ & $\begin{array}{l}\{6,17,38\} \\
\{41,55,25\} \\
\{44,31\}\end{array}$ & $\begin{array}{l}\{7,18,39\} \\
\{19,34,54\} \\
\{24,52\}\end{array}$ \\
\hline$t=8, u=65$ & $\begin{array}{l}\{0,31,23\} \\
\{10,26,30\} \\
\{16,45,36\} \\
\{62,46\}\end{array}$ & $\begin{array}{l}\{1,25,11\} \\
\{15,47,32\} \\
\{39,64,51\} \\
\{38,60\}\end{array}$ & $\begin{array}{l}\{2,20,13\} \\
\{12,33,40\} \\
\{53,63\} \\
\{54,57\}\end{array}$ & $\begin{array}{l}\{3,35,9\} \\
\{18,21,22\} \\
\{7,48\}\end{array}$ & $\begin{array}{l}\{4,34,6\} \\
\{19,42,50\} \\
\{52,59\}\end{array}$ & $\begin{array}{l}\{5,24,27\} \\
\{28,55,44\} \\
\{8,43\}\end{array}$ & $\begin{array}{l}\{14,41,29\} \\
\{37,56,61\} \\
\{17,49\}\end{array}$ \\
\hline & $\begin{array}{l}\{0,2,28\} \\
\{9,22,39\} \\
\{41,47,58\} \\
\{19,57\}\end{array}$ & $\begin{array}{l}\{1,15,33\} \\
\{14,16,29\} \\
\{38,56,61\} \\
\{12,52\}\end{array}$ & $\begin{array}{l}\{3,23,11\} \\
\{13,35,44\} \\
\{49,54\} \\
\{42,60\}\end{array}$ & $\begin{array}{l}\{4,20,26\} \\
\{18,48,21\} \\
\{64,30\}\end{array}$ & $\begin{array}{l}\{6,27,31\} \\
\{25,32,46\} \\
\{45,51\}\end{array}$ & $\begin{array}{l}\{7,17,8\} \\
\{24,53,43\} \\
\{63,50\}\end{array}$ & $\begin{array}{l}\{5,10,34\} \\
\{36,62,37\} \\
\{40,59\}\end{array}$ \\
\hline & $\begin{array}{l}\{0,31,23\} \\
\{10,26,30\} \\
\{16,45,36\} \\
\{43,60\}\end{array}$ & $\begin{array}{l}\{1,25,11\} \\
\{15,47,32\} \\
\{39,64,51\} \\
\{53,8\}\end{array}$ & $\begin{array}{l}\{2,20,13\} \\
\{12,33,40\} \\
\{63,52\} \\
\{46,7\}\end{array}$ & $\begin{array}{l}\{3,35,9\} \\
\{18,21,22\} \\
\{62,48\}\end{array}$ & $\begin{array}{l}\{4,34,6\} \\
\{19,42,50\} \\
\{49,57\}\end{array}$ & $\begin{array}{l}\{5,24,27\} \\
\{28,55,44\} \\
\{38,59\}\end{array}$ & $\begin{array}{l}\{14,41,29\} \\
\{37,56,61\} \\
\{54,58\}\end{array}$ \\
\hline & $\begin{array}{l}\{0,2,28\} \\
\{9,22,39\} \\
\{41,47,58\} \\
\{59,30\}\end{array}$ & $\begin{array}{l}\{1,15,33\} \\
\{14,16,29\} \\
\{38,56,61\} \\
\{54,63\}\end{array}$ & $\begin{array}{l}\{3,23,11\} \\
\{13,35,44\} \\
\{55,57\} \\
\{40,52\}\end{array}$ & $\begin{array}{l}\{4,20,26\} \\
\{18,48,21\} \\
\{42,19\}\end{array}$ & $\begin{array}{l}\{6,27,31\} \\
\{25,32,46\} \\
\{50,51\}\end{array}$ & $\begin{array}{l}\{7,17,8\} \\
\{24,53,43\} \\
\{45,60\}\end{array}$ & $\begin{array}{l}\{5,10,34\} \\
\{36,62,37\} \\
\{49,12\}\end{array}$ \\
\hline$t=11, u=89$ & $\begin{array}{l}\{0,26,32\} \\
\{6,45,37\} \\
\{25,64,52\} \\
\{63,82,67\} \\
\{85,66\}\end{array}$ & $\begin{array}{l}\{1,19,42\} \\
\{8,50,43\} \\
\{13,53,56\} \\
\{68,84\} \\
\{65,87\}\end{array}$ & $\begin{array}{l}\{2,38,22\} \\
\{9,18,29\} \\
\{36,72,49\} \\
\{80,57\} \\
\{81,60\}\end{array}$ & $\begin{array}{l}\{3,14,33\} \\
\{16,40,41\} \\
\{30,58,70\} \\
\{74,79\} \\
\{83,73\}\end{array}$ & $\begin{array}{l}\{4,39,35\} \\
\{11,55,21\} \\
\{15,44,47\} \\
\{71,86\} \\
\{54,88\}\end{array}$ & $\begin{array}{l}\{5,12,20\} \\
\{24,46,48\} \\
\{23,61,28\} \\
\{69,77\}\end{array}$ & $\begin{array}{l}\{7,34,51\} \\
\{17,31,59\} \\
\{62,10,78\} \\
\{76,27\}\end{array}$ \\
\hline & $\begin{array}{l}\{0,1,25\} \\
\{7,24,50\} \\
\{33,63,76\} \\
\{60,77,66\} \\
\{80,83\}\end{array}$ & $\begin{array}{l}\{2,30,20\} \\
\{8,48,52\} \\
\{43,46,65\} \\
\{82,35\} \\
\{61,81\}\end{array}$ & $\begin{array}{l}\{3,44,39\} \\
\{12,41,49\} \\
\{18,55,32\} \\
\{74,85\} \\
\{67,14\}\end{array}$ & $\begin{array}{l}\{4,13,34\} \\
\{15,47,53\} \\
\{37,64,71\} \\
\{73,23\} \\
\{68,10\}\end{array}$ & $\begin{array}{l}\{6,28,40\} \\
\{11,26,36\} \\
\{69,70,22\} \\
\{75,27\} \\
\{59,87\}\end{array}$ & $\begin{array}{l}\{5,19,21\} \\
\{16,54,45\} \\
\{17,56,38\} \\
\{58,88\}\end{array}$ & $\begin{array}{l}\{9,29,42\} \\
\{31,57,62\} \\
\{51,84,86\} \\
\{79,72\}\end{array}$ \\
\hline & $\begin{array}{l}\{0,26,32\} \\
\{6,45,37\} \\
\{25,64,52\} \\
\{63,82,67\} \\
\{79,27\}\end{array}$ & $\begin{array}{l}\{1,19,42\} \\
\{8,50,43\} \\
\{13,53,56\} \\
\{81,68\} \\
\{57,86\}\end{array}$ & $\begin{array}{l}\{2,38,22\} \\
\{9,18,29\} \\
\{36,72,49\} \\
\{76,77\} \\
\{54,87\}\end{array}$ & $\begin{array}{l}\{3,14,33\} \\
\{16,40,41\} \\
\{30,58,70\} \\
\{88,71\} \\
\{60,85\}\end{array}$ & $\begin{array}{l}\{4,39,35\} \\
\{11,55,21\} \\
\{15,44,47\} \\
\{83,65\} \\
\{84,75\}\end{array}$ & $\begin{array}{l}\{5,12,20\} \\
\{24,46,48\} \\
\{23,61,28\} \\
\{66,80\}\end{array}$ & $\begin{array}{l}\{7,34,51\} \\
\{17,31,59\} \\
\{62,10,78\} \\
\{73,69\}\end{array}$ \\
\hline & $\begin{array}{l}\{0,1,25\} \\
\{7,24,50\} \\
\{33,63,76\} \\
\{60,77,66\} \\
\{75,87\}\end{array}$ & $\begin{array}{l}\{2,30,20\} \\
\{8,48,52\} \\
\{43,46,65\} \\
\{58,82\} \\
\{79,81\}\end{array}$ & $\begin{array}{l}\{3,44,39\} \\
\{12,41,49\} \\
\{18,55,32\} \\
\{72,78\} \\
\{73,27\}\end{array}$ & $\begin{array}{l}\{4,13,34\} \\
\{15,47,53\} \\
\{37,64,71\} \\
\{74,23\} \\
\{67,10\}\end{array}$ & $\begin{array}{l}\{6,28,40\} \\
\{11,26,36\} \\
\{69,70,22\} \\
\{59,85\} \\
\{68,14\}\end{array}$ & $\begin{array}{l}\{5,19,21\} \\
\{16,54,45\} \\
\{17,56,38\} \\
\{88,61\}\end{array}$ & $\begin{array}{l}\{9,29,42\} \\
\{31,57,62\} \\
\{51,84,86\} \\
\{80,35\}\end{array}$ \\
\hline$t$ & $\begin{array}{l}\{27,28,75\} \\
\{1,9,40\} \\
\{14,23,42\} \\
\{5,15,49\} \\
\{67,95\} \\
\{91,6\}\end{array}$ & $\begin{array}{l}\{29,30,77\} \\
\{4,12,43\} \\
\{37,48,66\} \\
\{72,82,19\} \\
\{2,81\}\end{array}$ & $\begin{array}{l}\{31,32,79\} \\
\{34,50,74\} \\
\{44,55,73\} \\
\{47,59,92\} \\
\{93,3\}\end{array}$ & $\begin{array}{l}\{10,25,45\} \\
\{36,52,76\} \\
\{51,62,80\} \\
\{61,64\} \\
\{60,83\}\end{array}$ & $\begin{array}{l}\{11,26,46\} \\
\{38,54,78\} \\
\{56,69,86\} \\
\{65,85\} \\
\{21,63\}\end{array}$ & $\begin{array}{l}\{18,33,53\} \\
\{7,16,35\} \\
\{57,70,87\} \\
\{89,17\} \\
\{24,68\}\end{array}$ & $\begin{array}{l}\{0,8,39\} \\
\{13,22,41\} \\
\{58,71,88\} \\
\{90,96\} \\
\{84,94\}\end{array}$ \\
\hline & $\begin{array}{l}\{3,13,47\} \\
\{73,70,32\} \\
\{91,84,48\} \\
\{19,25,46\} \\
\{35,65\} \\
\{28,49\}\end{array}$ & $\begin{array}{l}\{5,17,50\} \\
\{74,71,33\} \\
\{87,89,15\} \\
\{30,36,57\} \\
\{66,34\}\end{array}$ & $\begin{array}{l}\{6,18,51\} \\
\{80,75,38\} \\
\{88,90,16\} \\
\{37,41,63\} \\
\{11,45\}\end{array}$ & $\begin{array}{l}\{7,21,53\} \\
\{81,76,39\} \\
\{92,94,20\} \\
\{61,1\} \\
\{93,10\}\end{array}$ & $\begin{array}{l}\{8,22,54\} \\
\{82,77,40\} \\
\{95,2,24\} \\
\{68,44\} \\
\{12,52\}\end{array}$ & $\begin{array}{l}\{9,23,55\} \\
\{85,78,42\} \\
\{0,4,26\} \\
\{67,96\} \\
\{27,58\}\end{array}$ & $\begin{array}{l}\{72,69,31\} \\
\{86,79,43\} \\
\{56,62,83\} \\
\{59,14\} \\
\{60,64\}\end{array}$ \\
\hline & $\begin{array}{l}\{27,28,75\} \\
\{1,9,40\} \\
\{14,23,42\} \\
\{5,15,49\} \\
\{68,6\} \\
\{85,24\}\end{array}$ & $\begin{array}{l}\{29,30,77\} \\
\{4,12,43\} \\
\{37,48,66\} \\
\{72,82,19\} \\
\{94,81\}\end{array}$ & $\begin{array}{l}\{31,32,79\} \\
\{34,50,74\} \\
\{44,55,73\} \\
\{47,59,92\} \\
\{90,2\}\end{array}$ & $\begin{array}{l}\{10,25,45\} \\
\{36,52,76\} \\
\{51,62,80\} \\
\{95,93\} \\
\{67,83\}\end{array}$ & $\begin{array}{l}\{11,26,46\} \\
\{38,54,78\} \\
\{56,69,86\} \\
\{20,3\} \\
\{65,84\}\end{array}$ & $\begin{array}{l}\{18,33,53\} \\
\{7,16,35\} \\
\{57,70,87\} \\
\{17,60\} \\
\{63,89\}\end{array}$ & $\begin{array}{l}\{0,8,39\} \\
\{13,22,41\} \\
\{58,71,88\} \\
\{91,64\} \\
\{96,21\}\end{array}$ \\
\hline
\end{tabular}

THE ELECTRONIC JOURNAL OF COMBINATORICS 11 (2004), \#R24 


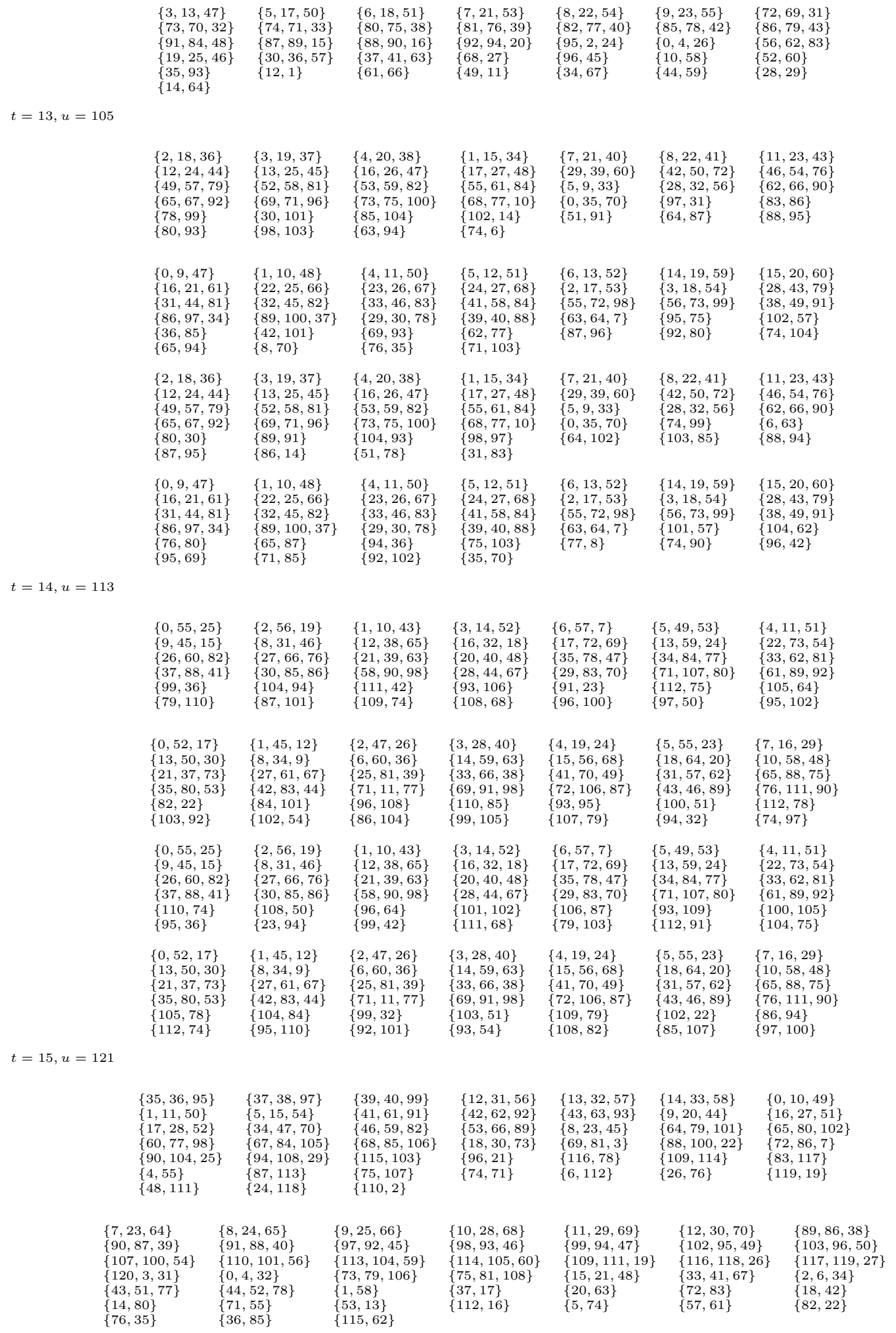

THE ELECTRONIC JOURNAL OF COMBINATORICS 11 (2004), \#R24 


\begin{tabular}{|c|c|c|c|c|c|c|}
\hline $\begin{array}{l}\{35,36,95\} \\
\{1,11,50\} \\
\{17,28,52\} \\
\{60,77,98\} \\
\{90,104,25\} \\
\{83,21\} \\
\{96,48\}\end{array}$ & $\begin{array}{l}\{37,38,97\} \\
\{5,15,54\} \\
\{34,47,70\} \\
\{67,84,105\} \\
\{94,108,29\} \\
\{75,117\} \\
\{107,113\}\end{array}$ & $\begin{array}{l}\{39,40,99\} \\
\{41,61,91\} \\
\{46,59,82\} \\
\{68,85,106\} \\
\{6,4\} \\
\{74,118\} \\
\{71,24\}\end{array}$ & $\begin{array}{l}\{12,31,56\} \\
\{42,62,92\} \\
\{53,66,89\} \\
\{18,30,73\} \\
\{116,26\} \\
\{111,76\}\end{array}$ & $\begin{array}{l}\{13,32,57\} \\
\{43,63,93\} \\
\{8,23,45\} \\
\{69,81,3\} \\
\{2,78\} \\
\{114,115\}\end{array}$ & $\begin{array}{l}\{14,33,58\} \\
\{9,20,44\} \\
\{64,79,101\} \\
\{88,100,22\} \\
\{103,110\} \\
\{109,119\}\end{array}$ & $\begin{array}{l}\{0,10,49\} \\
\{16,27,51\} \\
\{65,80,102\} \\
\{72,86,7\} \\
\{120,87\} \\
\{19,55\}\end{array}$ \\
\hline $\begin{array}{l}\{7,23,64\} \\
\{90,87,39\} \\
\{107,100,54\} \\
\{120,3,31\} \\
\{43,51,77\} \\
\{83,112\} \\
\{18,36\}\end{array}$ & $\begin{array}{l}\{8,24,65\} \\
\{91,88,40\} \\
\{110,101,56\} \\
\{0,4,32\} \\
\{44,52,78\} \\
\{57,35\} \\
\{58,72\}\end{array}$ & $\begin{array}{l}\{9,25,66\} \\
\{97,92,45\} \\
\{113,104,59\} \\
\{73,79,106\} \\
\{115,76\} \\
\{80,13\} \\
\{37,74\}\end{array}$ & $\begin{array}{l}\{10,28,68\} \\
\{98,93,46\} \\
\{114,105,60\} \\
\{75,81,108\} \\
\{42,14\} \\
\{85,55\}\end{array}$ & $\begin{array}{l}\{11,29,69\} \\
\{99,94,47\} \\
\{109,111,19\} \\
\{15,21,48\} \\
\{63,71\} \\
\{62,53\}\end{array}$ & $\begin{array}{l}\{12,30,70\} \\
\{102,95,49\} \\
\{116,118,26\} \\
\{33,41,67\} \\
\{61,84\} \\
\{1,20\}\end{array}$ & $\begin{array}{l}\{89,86,38\} \\
\{103,96,50\} \\
\{117,119,27\} \\
\{2,6,34\} \\
\{5,22\} \\
\{82,17\}\end{array}$ \\
\hline \multicolumn{7}{|l|}{$t=16, u=129$} \\
\hline $\begin{array}{l}\{2,22,44\} \\
\{12,28,52\} \\
\{54,66,92\} \\
\{74,80,109\} \\
\{91,93,124\} \\
\{117,36\} \\
\{82,123\}\end{array}$ & $\begin{array}{l}\{3,23,45\} \\
\{13,29,53\} \\
\{58,68,95\} \\
\{75,81,110\} \\
\{111,113,15\} \\
\{108,119\} \\
\{90,104\}\end{array}$ & $\begin{array}{l}\{4,24,46\} \\
\{16,30,55\} \\
\{59,69,96\} \\
\{77,83,112\} \\
\{105,116,34\} \\
\{39,5\} \\
\{79,114\}\end{array}$ & $\begin{array}{l}\{1,19,42\} \\
\{17,31,56\} \\
\{60,70,97\} \\
\{6,10,40\} \\
\{0,43,86\} \\
\{20,78\} \\
\{125,102\}\end{array}$ & $\begin{array}{l}\{7,25,48\} \\
\{18,32,57\} \\
\{63,71,99\} \\
\{33,37,67\} \\
\{85,94\} \\
\{126,107\} \\
\{35,62\}\end{array}$ & $\begin{array}{l}\{8,26,49\} \\
\{9,21,47\} \\
\{64,72,100\} \\
\{84,88,118\} \\
\{103,127\} \\
\{41,98\} \\
\{121,122\}\end{array}$ & $\begin{array}{l}\{11,27,51\} \\
\{38,50,76\} \\
\{65,73,101\} \\
\{87,89,120\} \\
\{61,106\} \\
\{115,14\}\end{array}$ \\
\hline $\begin{array}{l}\{0,11,58\} \\
\{18,25,74\} \\
\{23,42,86\} \\
\{41,56,102\} \\
\{108,121,44\} \\
\{70,117\} \\
\{95,64\}\end{array}$ & $\begin{array}{l}\{1,12,59\} \\
\{2,7,57\} \\
\{24,43,87\} \\
\{45,60,106\} \\
\{89,90,20\} \\
\{123,71\} \\
\{128,79\}\end{array}$ & $\begin{array}{l}\{4,13,61\} \\
\{21,26,76\} \\
\{34,53,97\} \\
\{48,69,101\} \\
\{109,110,40\} \\
\{122,125\} \\
\{120,46\}\end{array}$ & $\begin{array}{l}\{5,14,62\} \\
\{22,27,77\} \\
\{19,36,81\} \\
\{51,72,104\} \\
\{118,119,49\} \\
\{112,75\} \\
\{78,111\}\end{array}$ & $\begin{array}{l}\{6,15,63\} \\
\{28,31,82\} \\
\{37,54,99\} \\
\{52,73,105\} \\
\{91,93\} \\
\{113,92\} \\
\{98,8\}\end{array}$ & $\begin{array}{l}\{9,16,65\} \\
\{29,32,83\} \\
\{38,55,100\} \\
\{67,80,3\} \\
\{68,124\} \\
\{47,107\} \\
\{94,114\}\end{array}$ & $\begin{array}{l}\{10,17,66\} \\
\{30,33,84\} \\
\{35,50,96\} \\
\{103,116,39\} \\
\{88,126\} \\
\{115,85\}\end{array}$ \\
\hline $\begin{array}{l}\{2,22,44\} \\
\{12,28,52\} \\
\{54,66,92\} \\
\{74,80,109\} \\
\{91,93,124\} \\
\{35,78\} \\
\{36,103\}\end{array}$ & $\begin{array}{l}\{3,23,45\} \\
\{13,29,53\} \\
\{58,68,95\} \\
\{75,81,110\} \\
\{111,113,15\} \\
\{104,121\} \\
\{90,14\}\end{array}$ & $\begin{array}{l}\{4,24,46\} \\
\{16,30,55\} \\
\{59,69,96\} \\
\{77,83,112\} \\
\{105,116,34\} \\
\{125,79\} \\
\{127,5\}\end{array}$ & $\begin{array}{l}\{1,19,42\} \\
\{17,31,56\} \\
\{60,70,97\} \\
\{6,10,40\} \\
\{0,43,86\} \\
\{115,119\} \\
\{98,85\}\end{array}$ & $\begin{array}{l}\{7,25,48\} \\
\{18,32,57\} \\
\{63,71,99\} \\
\{33,37,67\} \\
\{108,82\} \\
\{106,62\} \\
\{126,61\}\end{array}$ & $\begin{array}{l}\{8,26,49\} \\
\{9,21,47\} \\
\{64,72,100\} \\
\{84,88,118\} \\
\{114,122\} \\
\{94,123\} \\
\{107,20\}\end{array}$ & $\begin{array}{l}\{11,27,51\} \\
\{38,50,76\} \\
\{65,73,101\} \\
\{87,89,120\} \\
\{41,102\} \\
\{128,39\}\end{array}$ \\
\hline $\begin{array}{l}\{0,11,58\} \\
\{18,25,74\} \\
\{23,42,86\} \\
\{41,56,102\} \\
\{108,121,44\} \\
\{95,117\} \\
\{113,88\}\end{array}$ & $\begin{array}{l}\{1,12,59\} \\
\{2,7,57\} \\
\{24,43,87\} \\
\{45,60,106\} \\
\{89,90,20\} \\
\{70,85\} \\
\{125,115\}\end{array}$ & $\begin{array}{l}\{4,13,61\} \\
\{21,26,76\} \\
\{34,53,97\} \\
\{48,69,101\} \\
\{109,110,40\} \\
\{79,91\} \\
\{71,107\}\end{array}$ & $\begin{array}{l}\{5,14,62\} \\
\{22,27,77\} \\
\{19,36,81\} \\
\{51,72,104\} \\
\{118,119,49\} \\
\{78,8\} \\
\{123,128\}\end{array}$ & $\begin{array}{l}\{6,15,63\} \\
\{28,31,82\} \\
\{37,54,99\} \\
\{52,73,105\} \\
\{92,124\} \\
\{75,93\} \\
\{127,111\}\end{array}$ & $\begin{array}{l}\{9,16,65\} \\
\{29,32,83\} \\
\{38,55,100\} \\
\{67,80,3\} \\
\{120,126\} \\
\{122,68\} \\
\{64,114\}\end{array}$ & $\begin{array}{l}\{10,17,66\} \\
\{30,33,84\} \\
\{35,50,96\} \\
\{103,116,39\} \\
\{46,112\} \\
\{47,98\}\end{array}$ \\
\hline
\end{tabular}

\section{A.6 HCURFs of type $8^{21 t+1}$}

An $\mathrm{HCURF}_{8}$ of type $1^{22}$ with partition $2^{9} 3^{1}(\mathrm{t}=1)$

$\begin{array}{rlllll}12: & \{0,1,3\} & \{2,13\} & \{4,8\} & \{5,9\} & \{6,10\} \\ & \{7,11\} & \{14,18\} & \{15,19\} & \{16,20\} & \{17,21\} \\ 2: & \{0,1,3\} & \{4,9\} & \{5,10\} & \{6,11\} & \{7,8\} \\ & \{12,17\} & \{13,18\} & \{14,19\} & \{15,20\} & \{16,21\} \\ 5: & \{0,1,3\} & \{2,18\} & \{4,20\} & \{6,12\} & \{7,13\} \\ & \{8,14\} & \{9,15\} & \{10,16\} & \{11,17\} & \{19,21\} \\ 19: & \{0,1,3\} & \{2,13\} & \{4,12\} & \{5,20\} & \{6,21\} \\ & \{7,14\} & \{8,15\} & \{9,16\} & \{10,17\} & \{11,18\} \\ 4: & \{0,1,3\} & \{2,5\} & \{6,14\} & \{7,15\} & \{8,16\} \\ & \{9,17\} & \{10,18\} & \{11,19\} & \{12,20\} & \{13,21\} \\ 4: & \{0,1,3\} & \{2,13\} & \{5,14\} & \{6,15\} & \{7,16\} \\ & \{8,17\} & \{9,18\} & \{10,19\} & \{11,20\} & \{12,21\} \\ 12: & \{0,1,3\} & \{2,13\} & \{4,14\} & \{5,15\} & \{6,16\} \\ & \{7,17\} & \{8,18\} & \{9,19\} & \{10,20\} & \{11,21\}\end{array}$


An $\mathrm{HCURF}_{8}$ of type $1^{43}$ with partition $2^{18} 3^{2}(\mathrm{t}=2)$

$\begin{array}{rlllll}24: & \{0,1,3\} & \{2,6,11\} & \{29,39\} & \{42,25\} & \{38,7\} \\ & \{37,22\} & \{10,28\} & \{16,36\} & \{12,31\} & \{19,27\} \\ & \{20,30\} & \{33,9\} & \{13,34\} & \{15,26\} & \{40,8\} \\ 33: & \{32,4\} & \{14,15\} & \{18,23\} & \{21,41\} & \{5,17\} \\ & \{10,26\} & \{2,6,11\} & \{42,24\} & \{41,16\} & \{25,38\} \\ & \{31,7\} & \{34,8\} & \{27,5\} & \{37,18\} & \{12,20\} \\ 21: & \{29,40\} & \{13,28\} & \{39,15\} & \{14,22\} & \{4,21\} \\ & \{0,1,3\} & \{2,6,11\} & \{15,22\} & \{35,37\} & \{19,36\} \\ & \{28,41\} & \{39,4\} & \{9,27\} & \{36,5\} & \{18,31\} \\ & \{20,40\} & \{38,14\} & \{7,10\} & \{16,33\} & \{17,32\} \\ & \{30,42\} & \{34,12\} & \{19,29\} & \{8,25\} & \{26,13\} \\ 31: & \{0,1,3\} & \{2,6,11\} & \{41,7\} & \{15,27\} & \{12,28\} \\ & \{9,29\} & \{24,39\} & \{8,21\} & \{36,30\} & \{25,35\} \\ & \{23,37\} & \{14,22\} & \{4,16\} & \{20,38\} & \{10,17\} \\ & \{34,5\} & \{42,19\} & \{26,40\} & \{32,13\} & \{18,33\} \\ 38: & \{0,1,3\} & \{2,6,11\} & \{12,23\} & \{27,37\} & \{13,19\} \\ & \{10,31\} & \{41,14\} & \{25,32\} & \{4,20\} & \{21,35\} \\ & \{42,9\} & \{22,16\} & \{17,24\} & \{40,5\} & \{30,36\} \\ & \{28,34\} & \{8,29\} & \{15,26\} & \{39,7\} & \{33,18\} \\ 9: & \{0,1,3\} & \{2,6,11\} & \{40,8\} & \{19,27\} & \{25,39\} \\ & \{5,15\} & \{32,16\} & \{17,24\} & \{7,20\} & \{29,33\} \\ & \{26,10\} & \{41,18\} & \{34,12\} & \{13,23\} & \{37,31\} \\ & \{21,35\} & \{30,4\} & \{42,28\} & \{38,14\} & \{36,22\} \\ 18: & \{0,1,3\} & \{2,6,11\} & \{8,33\} & \{31,9\} & \{17,30\} \\ & \{15,38\} & \{20,35\} & \{4,10\} & \{7,13\} & \{29,36\} \\ \{12,19\} & \{14,26\} & \{21,37\} & \{40,22\} & \{27,34\} \\ & \{32,24\} & \{25,41\} & \{28,39\} & \{5,23\} & \{42,16\}\end{array}$

\section{References}

[1] F. Bennett. Personal Communication, 2002.

[2] C. J. Colbourn and J. H. Dinitz, editors. The CRC Handbook of Combinatorial Designs. CRC Press, Boca Raton, 1996.

[3] C. J. Colbourn, D. R. Stinson, and L. Zhu. More frames with block size four. J. Combin. Math. Combin. Comput., 23:3-19, 1997.

[4] P. Danziger and B. Stevens. Class-uniformly resolvable designs. J. Combin. Des., 9:79-99, 2001.

[5] P. Danziger and B. Stevens. $\mathrm{HCURF}_{4}$ of type $1^{9 t+1}$ for $1 \leq t \leq 99$. Technical report, Ryerson University, 2003.

[6] P. Danziger and B. Stevens. HCURFs of type $6^{5 t+1}$. Technical report, Ryerson University, 2003.

[7] P. Danziger and B. Stevens. Class-uniformly resolvable group divisible structures I: Resolvable group divisible designs. Electron. J. Combin., 11:R23, 2004. 
[8] S. Furino, Y. Miao, and J. Yin. Frames and resolvable designs. CRC Press, Boca Raton, FL, 1996.

[9] A. M. Hamel, W. H. Mills, R. C. Mullin, R. Rees, D. R. Stinson, and J. X. Yin. The spectrum of $\operatorname{pbd}\left(\left\{5, k^{*}\right\}, v\right)$ for $k=9,13$. Ars Combin., 36:7-26, 1993.

[10] D. L. Kreher and D. R. Stinson. Small group-divisible designs with block size four. J. Statist. Plann. Inference, 58(1):111-118, 1997.

[11] E. Lamken, R. Rees, and S. Vanstone. Class-uniformly resolvable pairwise balanced designs with block sizes two and three. Discrete Math., 92:197-209, 1991.

[12] R. Rees and D. Stinson. Frames with block size four. Canad. J. Math., 44:107-120, 1992.

[13] D. R. Stinson. Frames for Kirkman triple systems. Discrete Math., 65:87-94, 1987.

[14] D. Wevrick and S. A. Vanstone. Class-uniformly resolvable designs with block sizes 2 and 3. J. Combin. Des., 4:177-202, 1996.

[15] R. Wilson. An existence theory for pairwise balanced designs III: A proof of the existence conjectures. J. Combin. Theory. Ser. A, 18:71-79, 1975. 